\title{
Autophagosomes fuse to phagosomes and facilitate the degradation of apoptotic cells in Caenorhabditis elegans
}

3 Omar Peña-Ramos ${ }^{1}$, Lucia Chiao ${ }^{1}$, Xianghua Liu ${ }^{1,2}$, Tianyou Yao ${ }^{1}$, Henry He ${ }^{1,3}$, and Zheng Zhou ${ }^{1,4}$

$4 \quad{ }^{1}$ Verna and Marrs McLean Department of Biochemistry and Molecular Biology, Baylor College of

5 Medicine, Houston, TX 77030

$6 \quad{ }^{2}$ Current address: Caris Life Sciences, Inc., Tempe, AZ 85281

$7 \quad{ }^{3}$ Current address: Department of Neurology Residency Program, University of Texas Southwestern

8 Medical Center, Dallas, TX 75390

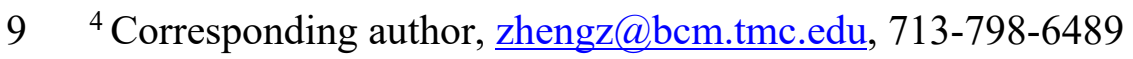

\section{Abstract}

12 Autophagosomes are double-membrane intracellular vesicles that degrade protein aggregates,

13 intracellular organelles, and other cellular components. During the embryonic development of the

14 nematode Caenorhabditis elegans, 113 somatic cells undergo apoptosis. These cells are engulfed and degraded by their neighboring cells. We discovered a novel role of autophagosomes in facilitating the degradation of apoptotic cells in C. elegans embryos using a real-time imaging technique. Specifically, the double-membrane autophagosomes in engulfing cells are recruited to the surfaces of phagosomes containing apoptotic cells and subsequently fuse to phagosomes, allowing the inner vesicle to enter the phagosomal lumen. Mutants defective in the production of autophagosomes display significant defects in the degradation of apoptotic cells, demonstrating the important contribution of autophagosomes to this process. The signaling pathway led by the phagocytic receptor CED-1, the adaptor protein CED-6, and the large GTPase dynamin (DYN-1) promotes the recruitment of autophagosomes to phagosomes. Moreover, the subsequent fusion of autophagosomes with phagosomes requires the functions of the small GTPase RAB-7 and the HOPS complex components. Our findings reveal that, unlike the singlemembrane, LC3-associated phagocytosis (LAP) vesicles reported for mammalian phagocytes, the canonical double-membrane autophagosomes facilitate the clearance of $C$. elegans apoptotic cells. These findings add autophagosomes to the collection of intracellular organelles that contribute to phagosome maturation, identify novel crosstalk between the autophagy and phagosome maturation pathways, and discover the upstream factors that initiate this crosstalk. 


\section{Introduction}

During metazoan development and adulthood, a large number of cells undergo apoptosis or cell suicide; these dying cells are engulfed by phagocytes and degraded inside phagosomes, vacuoles composed of the lipid bilayers originated from the plasma membrane [1,2]. Swift engulfment and degradation of apoptotic cells are critical for tissue remodeling, the resolution of the wound area, and the prevention and suppression of harmful inflammatory and autoimmune responses induced by the contest of the dying cells [2]. Critical to the degradation of phagosomal contents is the fusion of intracellular organelles, including lysosomes and early endosomes, to phagosomes, which results in the delivery of the content of these organelles to the phagosomal lumen [3]. Lysosomes, which contribute many kinds of hydrolytic enzymes, including proteases, nucleases, lipases, and hydrolyzing enzymes for polysaccharides to the lumen of phagosomes, are the most pivotal organelles that support phagosomal degradation [3]. The fusion of lysosomes to phagosomes also helps acidify the phagosomal lumen, creating a low $\mathrm{pH}$ condition in which the digestive enzymes are active [3]. Besides lysosomes and endosomes, whether other kinds of intracellular organelles fuse to phagosomes and contribute to the degradation of the apoptotic cells inside remains elusive.

Mammalian microtubule-associated protein 1 light $\underline{\text { chain }} \underline{3}$ (MAP1-LC3, or LC3) protein is a member of the ATG8 protein family [4]. LC3 molecules that are conjugated to the lipid phosphatidylethanolamine (PE) are most often observed on the surfaces of autophagosomes, doublemembrane organelles that are a key structure of macroautophagy [4]. In fact, LC3 is a well-established marker for autophagosomes [4]. In mammalian cells, lipidated LC3 molecules were also reported to label a novel kind of vesicles referred to as LC3-associated phagocytosis (LAP) vesicles, which are single-membrane vesicles [5]. LAP vesicles were reported to be the third kind of intracellular vesicles that fuse to phagosomes and facilitate the degradation of apoptotic cells in mice [6-8].

Autophagy is an evolutionarily conserved cellular event that plays an essential role in maintaining cellular homeostasis by enveloping harmful protein aggregates and damaged cellular organelles in double-membrane autophagosomes and subsequently degrading them via fusion with lysosomes [9]. Autophagy also supports cell survival during nutrient starvation by capturing intracellular organelles into autophagosomes and converting them to nutrients and energy sources [9]. Autophagosome formation requires the organized action of a set of proteins known as autophagic related (ATG) proteins. It is a process of three sequential steps: initiation, nucleation, and expansion, until an autophagosome fully forms and closes [10]. After formation, autophagosomes undergo a maturation process through fusion with lysosomes, which provide digestive enzymes to degrade 
autophagosomal contents [10]. In the nematode C. elegans, several autophagy genes have been reported to facilitate the clearance of apoptotic cells [11-13]. However, it is unknown whether autophagosome, as a particular type of cellular organelle, is involved in the clearance of apoptotic cells or whether these atg genes have additional functions, such as forming LAP vesicles.

Although both are labeled with LC3, mammalian LAP vesicles and autophagosomes are different in several key aspects. First, LAP vesicles are single-membrane vesicles, unlike autophagosomes, which are double-membrane vesicles [5,10]. In addition, although the formation of LAP vesicles relies on a lot of autophagy genes, $u l k 1$, $\operatorname{atg} 13$, and $\operatorname{atg} 14$, three genes whose products act in the initiation complexes for autophagosomes, are dispensable for the generation of LAP vesicles $[5,7,14]$. During the initiation of autophagosomes formation, which starts with the appearance of a membrane structure known as a phagophore, ULK1, a serine-threonine kinase, forms a protein complex with ATG13 and two other proteins and phosphorylates the class-III phosphoinositide 3-kinase (PI3K) VPS34 as well as the rest of the VPS34 complex (ATG6, ATG14, and VPS15), triggering the production of PtdIns(3)P on the phagosphore [10]. These distinct features of autophagosomes and LAP vesicles are critical for distinguishing whether an LC3-labeled vesicle is an autophagosome or a LAP vesicle.

During C. elegans embryonic development, 131 somatic cells undergo apoptosis and are swiftly engulfed and degraded by neighboring cells $[15,16]$. Apoptotic cells display a "button-like" structure under the Differential Contrast Interference (DIC) microscopy and are referred to as cell corpses $[15,16]$. Mutants defective in the clearance of cell corpses exhibit an increased number of persistent cell corpses, a phenotype known as cell death abnormal (Ced) [17]. Previous genetic studies revealed two parallel, partially redundant pathways that primarily drive the clearance of $C$. elegans cell corpses. These include a signaling pathway led by CED-1, a phagocytic receptor for apoptotic cells, CED-6, an adaptor protein for CED-1, and DYN-1, a large GTPase playing many roles in membrane trafficking, and the other pathway led by the small Rac1 GTPase CED-10, and CED-5 and CED-12, the bipartite nucleotide exchange factor for CED-10 [18]. Unlike the CED-10 pathway, which primarily regulates cell corpse engulfment, the CED-1 pathway has two separate functions, regulating both the engulfment and degradation of cell corpses [19]. CED-1 on neighboring engulfing cells recognizes the "eat me" signal on the surfaces of cell corpses and is enriched to the region of the plasma membrane facing a cell corpse [20]. This enrichment initiates the extension of pseudopods along the cell corpse and the subsequent closure of the phagocytic cup to form a nascent phagosome [21,22]. Moreover, CED-1 also plays a distinct role in initiating the degradation of phagosomal contents [19]. CED-1 remains transiently 
enriched on the surface of nascent phagosomes, where it facilitates the CED-6 and DYN-1-mediated sequential recruitment of the Class II phosphatidylinositol 3-bisphosphate (PtdIns(3)P) kinase PIKI-1, the Class III PtdIns(3)P kinase VPS-34, and the small GTPases RAB-5 and RAB-7 to phagosomal surfaces $[19,23]$. The robustly produced PtdIns(3)P and the RAB-5 and RAB-7 GTPases further recruit effectors for PtdIns(3)P and the RAB proteins, respectively, which drive the recruitment and fusion of early endosomes and lysosomes to a phagosome, leading to the degradation of the cell corpse inside [2325]. RAB-7, in particular, is responsible for the fusion of lysosomes to phagosomes [19].

Autophagy and phagocytosis are two distinct lysosomal-mediated cellular degradation pathways designated to eliminate intracellular and extracellular components, respectively. Previously, whether canonical autophagosomes were involved in the degradation of phagosomal contents was unknown. We report here that, in C. elegans embryos, during the maturation of phagosomes that contain apoptotic cells, LC3-labeled, double-membrane canonical autophagosomes are recruited to phagosomal surfaces and subsequently fuse to these phagosomes. We have further discovered that this event facilitates the degradation of apoptotic cells inside the phagosomal lumen and is driven by the signaling pathway led by CED-1. This autophagosome-phagosome fusion represents a novel mechanism that contributes to the degradation of phagosomal contents.

\section{Results}

\section{Vesicles labeled with GFP-tagged LC3 are recruited to the surfaces of phagosomes}

The ATG8 protein family is composed of two subfamilies, the LC3 subfamily, and the

GABARAP subfamily, which are very close to each other in sequence; the ATG8 family is also referred to as the LC3/GABARAP family [4]. C. elegans has two LC3/GABARAP family members, LGG-1 and LGG-2, which belong to the LC3 and GABARAP subfamilies, respectively (Fig 1A) [26]. Both LGG-1 and LGG-2 are attached to autophagosomes, except that they each label autophagosomes of different maturity [26].

To determine whether autophagosomes interact with phagosomes that contain apoptotic cells in C. elegans embryos, we constructed the GFP-tagged LGG-1 and LGG-2 reporters that were expressed under the control of the ced-1 promoter $\left(\mathrm{P}_{c e d-1}\right)$, a well-documented engulfing cell-specific promoter [20,27]. In embryos, we observed numerous GFP::LGG-1 ${ }^{+}$and GFP::LGG-2 ${ }^{+}$puncta (Fig 1, D,G). Using our previously established time-lapse recording protocol [27], we observed the enrichment of GFP::LGG- $1^{+}$and GFP-LGG-2 ${ }^{+}$puncta to the surfaces of the phagosomes, including the phagosomes 
126 containing apoptotic cells C1, C2, and C3 (Fig 1 B, D, G). These GFP-labeled puncta were not 127 observed inside the phagosomal lumen (Fig 1 D, G, top panels).

128 C. elegans LGG-1 and LGG-2 were both reported to specifically attach to autophagosomes 129 through their lipid tails [26,28]. To verify that the observed GFP puncta are LGG-labeled lipid vesicles 130 and not artifacts of protein aggregation, we tested two mutant constructs, GFP::LGG-1(G116A) and 131 GFP::LGG-2(G130A), which bear mutations in the lipidation sites of the LGG proteins and are deficient 132 for membrane targeting [26]. We found that both GFP::LGG-1(G116A) and GFP::LGG-2(G130A) 133 display a diffuse cytosolic localization pattern (Fig 1 D, G, bottom panels), in stark contrast to the 134 punctate pattern presented by GFP::LGG-1 and GFP::LGG-2. This result indicates that GFP::LGG-1 135 and GFP::LGG-2 are membrane attached and thus label lipid vesicles.

\section{The LGG-tagged puncta that fuse to phagosomes are double-membrane vesicles}

The fluorophore within GFP is sensitive to acidic $\mathrm{pH}(\mathrm{pKa}=6.0)$ [29], thus its signal diminishes when GFP is inside the acidic lumen of lysosomes and phagosomes. To further monitor the fate of the $\mathrm{LGG}^{+}$puncta after they are recruited to the surfaces of phagosomes, we replaced GFP with mCherry $(\mathrm{pKa}<4.5)$ or $\mathrm{mNeonGreen}(\mathrm{mNG})(\mathrm{pKa}=5.1)$, both of which are more resistant than GFP to the acidic $\mathrm{pH}$ environment [30,31], allowing fluorescence signal inside the phagosomal lumen to be detected. Coexpressed with the mCherry::LGG or mNG::LGG reporter are the CED-1::GFP or PH(hPLC $\gamma):: m R F P$ reporters, which were used in the time-lapse experiments as markers for the extending pseudopods, allowing us to determine the moment when the pseudopods sealed up and a nascent phagosome was born (Fig 1 E, F, H, I, top panels). In time-lapse image series of the clearance of C1, C2, and C3, we found that like the GFP::LGG reporters, the mCherry::LGG and $\mathrm{mNG::LGG} \mathrm{reporters} \mathrm{were} \mathrm{enriched} \mathrm{on}$ the surfaces of phagosomes; moreover, unlike the GFP::LGG reporters, the mCherry and mNG tagged reporters subsequently entered phagosomal lumen (Fig 1 E, F, H, I). The fluorescence signal intensity increases over time with the continuous recruitment of the $\mathrm{LGG}^{+}$puncta on phagosomal surfaces (Fig 1 E, F, H, I, Movie S1, S2, and S3). We measured the intensity of the mCherry::LGG-1 and mCherry::LGG-2 signal in the center of the C3 phagosomes over time from the start of the phagosome (Materials and Methods) (Fig 1C) and observed over 10-fold increases of the signal intensity within $60 \mathrm{~min}$ (Fig 1J). The appearance of the membrane-attached mCherry::LGG and mNG::LGG signal in the phagosomal lumen indicates that the $\mathrm{LGG}^{+}$vesicles fuse with the phagosomal membrane (Fig 1K).

They further suggest that these vesicles are composed of double membranes labeled with the LGG reporter molecules on both the outer and inner membranes (Fig 1K). If these $\mathrm{LGG}^{+}$vesicles were 
158 single-membrane, no membrane-attached LGG reporter would end up inside the phagosomal lumen 159 because, as a result of fusion, the reporter molecules will be retained on the phagosomal membrane (Fig 160 1K). Indeed, when CTNS-1, a lysosomal transmembrane protein, is tagged with mRFP, which is acid161 resistant $(\mathrm{pKa}=4.5$ [30]), on its C-terminus, the lysosome-phagosome fusion event resulted in the 162 incorporation of the mRFP signal to the phagosomal membrane; as a result, the CTNS-1::mRFP signal is 163 only observed on the phagosomal surface, not in the phagosomal lumen (Fig S1) [19]. 
A<smiles>[Mg][Mg]</smiles>

LGG-1

LGG-2 1

D

GFP::LGG-1

GFP::LGG-1

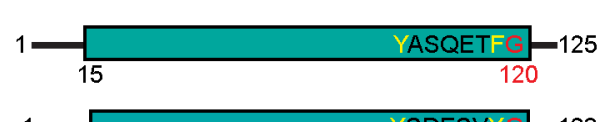

E

GFP::CED-1

mCherry

:LGG-1

F

$\mathrm{PH}(\mathrm{PLC} \delta)$ $\because \mathrm{mRFP}$

mNG::LGG-1

G

GFP::LGG-2

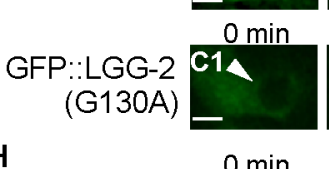

H

GFP::CED-1

mCherry

$\therefore$ LGG-2

$\mathrm{PH}(\mathrm{PLC} \delta)$
:.mRFP

$\mathrm{mNG}:$ :LGG-2
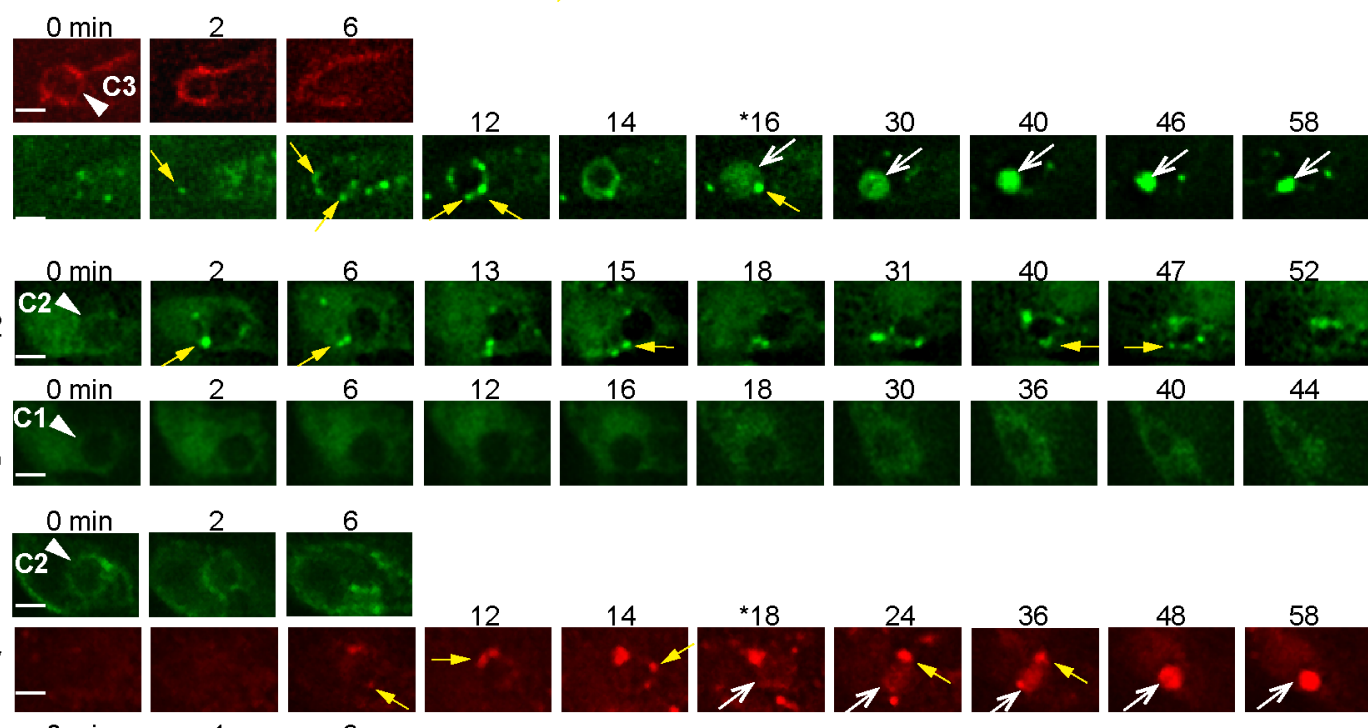

J

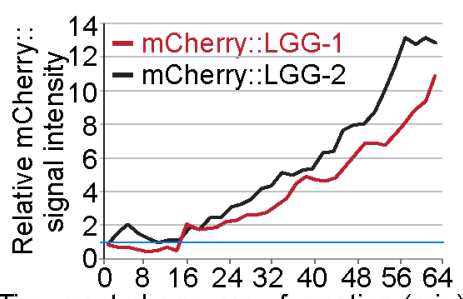

Time post phagosome formation (min)
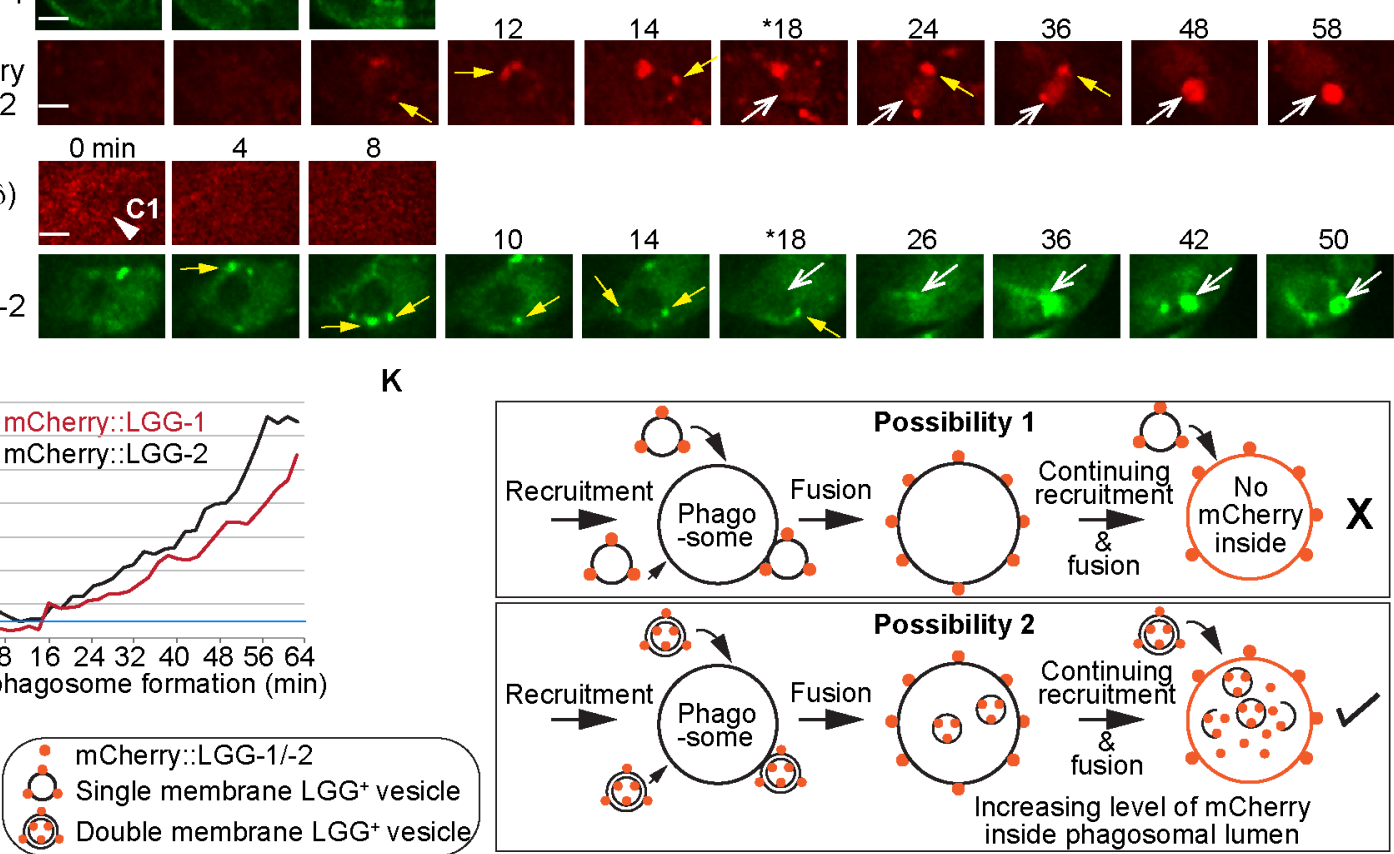

$\mathrm{K}$

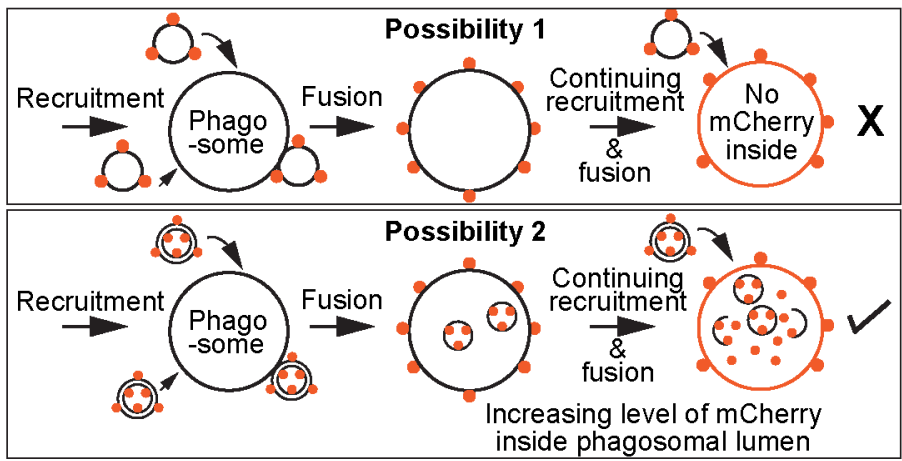

165

Figure 1. The vesicles labeled with LGG-1 or LGG-2 are recruited to the surface of phagosomes 
167 (A) Domain structures of mammalian LC3 and C. elegans LGG-1 and LGG-2. The green box indicates 168 the conserved ubiquitin-like domain. Residues in white are conserved among the three proteins. The 169 glycine residue in red is the site where the lipid tail is attached to.

170 (B) A diagram illustrating the three phagosomes that contain cell corpses $\mathrm{C} 1, \mathrm{C} 2$, and $\mathrm{C} 3$, with which 171 we monitor the dynamic recruitment and fusion of autophagosomes, at $\sim 330$ min post-the $1^{\text {st }}$ embryonic 172 division. Both the positions of $\mathrm{C} 1, \mathrm{C} 2$, and C3 (brown dots) and the identities of their engulfing cells are 173 shown.

174 (C) A diagram illustrating that the relative $\mathrm{mNG}$ signal in the center of a phagosome is measured over 175 time to create sub-figure $(\mathrm{J})$. At time point $\mathrm{T}$ (time after engulfment), the Relative signal intensity $\mathrm{T}_{\mathrm{T}}=$ 176 (Unit Intensity(phagosome center) $)_{T}-$ Unit Intensity (background) $)_{T}$ / (Unit Intensity(phagosome 177 center) $)_{\mathrm{T} 0}-$ Unit Intensity(background) $)_{\mathrm{T}}$ ).

178 (D-I) Time-lapse images of indicated reporters starting when a nascent phagosome (white arrowheads) 179 just formed (time point “0 min”). All reporters were expressed under the control of $\mathrm{P}_{\text {ced-1 }}$. Scale bars are $1802 \mu \mathrm{m}$. Solid white arrowheads label nascent phagosomes. Yellow arrows mark a few LGG-labeled 181 puncta on the surface of phagosomes. Open white arrows in $(E, F, H, I)$ label the phagosomal lumen in 182 which the LGG signal is observed. "*” is the time point when the LGG signal is first seen inside the 183 phagosomal lumen. CED-1::GFP $(E, H)$ and PH(PLCY)::mRFP $(F, I)$ are co-expressed markers that 184 label the surfaces of nascent phagosomes.

185 (D) GFP::LGG-1-labeled puncta are observed on the surface of a C3 phagosome, but the GFP signal is 186 not seen inside the phagosomal lumen. No GFP::LGG-1(G116A)-labeled puncta are seen on the 187 surface of phagosomes.

188 (E-F) The mCherry::LGG-1 (E) and mNG::LGG-1 (F) puncta are observed on the surface of a C3 189 phagosome and subsequently accumulate inside the phagosome lumen.

190 (G) GFP::LGG-2-labeled puncta are observed to attach on the surface of a C2 phagosome, but the 191 GFP signal does not enter the phagosomal lumen, whereas no GFP::LGG-1(G130A)-labeled puncta 192 are seen on the surface of phagosomes.

$193(\mathrm{H}-\mathrm{I})$ The mCherry::LGG-2 $(\mathrm{H})$ and mNG::LGG-2 $(\mathrm{I})$ puncta are observed on the surface of a C2 $(\mathrm{H})$ or 194 C1 (I) phagosome, respectively, and subsequently accumulate inside the phagosome lumen.

195 (J) The relative mCherry::LGG-1 or -2 signal intensity in the center of a phagosome (Y-axis) over time 196 (in 2-min interval) (X-axis). "0 min" indicates the moment when a nascent phagosome just formed. One 197 blue horizontal line indicates value "1", where no signal enrichment above background level is 198 observed.

199 (K) A diagram illustrating that those double membrane-vesicles labeled with mCherry::LGG on their 200 outer and inner membranes are recruited to phagosomal surfaces and fused to the phagosomal 201 membrane. After the fusion between the outer membrane of these vesicles and phagosomal 
membrane, the mCherry::LGG-tagged inner membrane enters the phagosomal lumen. The continuing incorporation of these double-membrane vesicles to phagosomes increases the mCherry signal level in the phagosomal lumen, observed over time. If the LGG-1 or LGG-2-labeled vesicles are of a single membrane, no fluorescence signal is expected to enter the phagosomal lumen.

\section{The $\mathrm{LGG}^{+}$vesicles that are incorporated into phagosomes are canonical autophagosomes}

The observation that the $\mathrm{LGG}^{+}$vesicles incorporated into phagosomes are likely doublemembrane vesicles reminded us of canonical autophagosomes rather than LAP vesicles. To further determine whether these vesicles represent canonical autophagosomes, we examined whether loss-offunction mutations of atg-7, atg-13, and epg-8, which are defective for the biogenesis of autophagosomes [32,33], impaired the production of these vesicles. C. elegans atg-13 and epg- 8 encode homologs of mammalian ATG13 and ATG14, respectively, which are essential for the biogenesis of autophagosomes but dispensable for that of LAP vesicles (Introduction) [34]. atg-7 encodes a homolog of mammalian ATG7, a protein essential for conjugating a phospholipid tail onto the LC3 family proteins and required for the biogenesis of both autophagosomes and LAP vesicles [10,34]. We first scored whether the mCherry::LGG-1/-2 reporters were observed in the center of 15 phagosomes during the phagosome maturation process (Figs 1C and 2), which is indicative of the fusion of doublemembrane mCherry::LGG ${ }^{+}$vesicles to phagosomes. In wild-type embryos, the steady entry of mCherry into phagosomes over time (Fig $1 \mathbf{E}, \mathbf{H})$ results in the increase of the average mCherry::LGG-1 and LGG-2 intensities to 9.2- and 8.4-fold of that at 0 min time point, respectively, at 60 min after the formation of a phagosome (Fig $\mathbf{2} \mathbf{H}, \mathbf{J})$. However, in atg-7(bp411) [35] mutant embryos, hardly any mCherry signal was observed inside phagosomes (Fig 2 A, D, G-J). At 60 min after the phagosome formation, the average mCherry signal intensities were merely 1.2 and 1.4-fold at 0 min time point (Fig $2 \mathbf{H}, \mathbf{J})$. These observations indicate a lack of $\mathrm{LGG}^{+}$vesicles that fuse to phagosomes. In atg13(bp414) and epg-8(bp251) mutant embryos, similar observations were made except that the defects were slightly weaker (Fig 2 B, C, E, F, G-J).

We next examined whether the mCherry::LGG-1/-2+ vesicles are produced in cells of the atg-7, atg-9, atg-13, and epg-8 mutant embryos. In wild-type embryos at mid-embryonic developmental stages ( $\sim 330, \sim 350, \sim 400$ min post- $1^{\text {st }}$ embryonic cell division), numerous $m C h e r r y{ }^{+}$puncta were observed (Figs S2A, S3A). In the atg-7 mutant embryos, rarely any such puncta existed (Figs S2B, S3B), consistent with a previous report [32]. atg-9 encodes ATG-9, the only transmembrane protein in the core autophagy machinery [32,34]. ATG9 plays an essential role in the expansion of phagophore and the biogenesis of autophagosomes [10]. In atg-9(bp564) mutant embryos that expressed mNG::LGG-1 
235 or ::LGG-2, much fewer $\mathrm{mNG}^{+}$puncta that might represent autophagosomes were observed, and the

$236 \mathrm{mNG}$ reporters label large aggregates (Fig S4), consistent with a previous report [36]. In atg-13 and

237 epg-8 mutant embryos, the numbers of $\mathrm{mCherry}-\mathrm{LGG}^{+}$puncta were also significantly reduced (Figs S2

238 C-D, S3 C-D), suggesting that the atg-13(bp414) and epn-8(bp251) mutations severely impaired the

239 biogenesis of autophagosomes, a phenotype that is consistent with previous reports [32,33]. The drastic

240 reduction in the number of LGG-1/-2-labeled puncta in atg-7, atg-9, atg-13, and epg-8 loss-of-function

241 mutants strongly indicate that these puncta belong to canonical autophagosomes.

A

A

DIC

mCherry

B

mCherry

C

C
CED-1
GFFP

DIC

mCherry

$::$ LGG-1
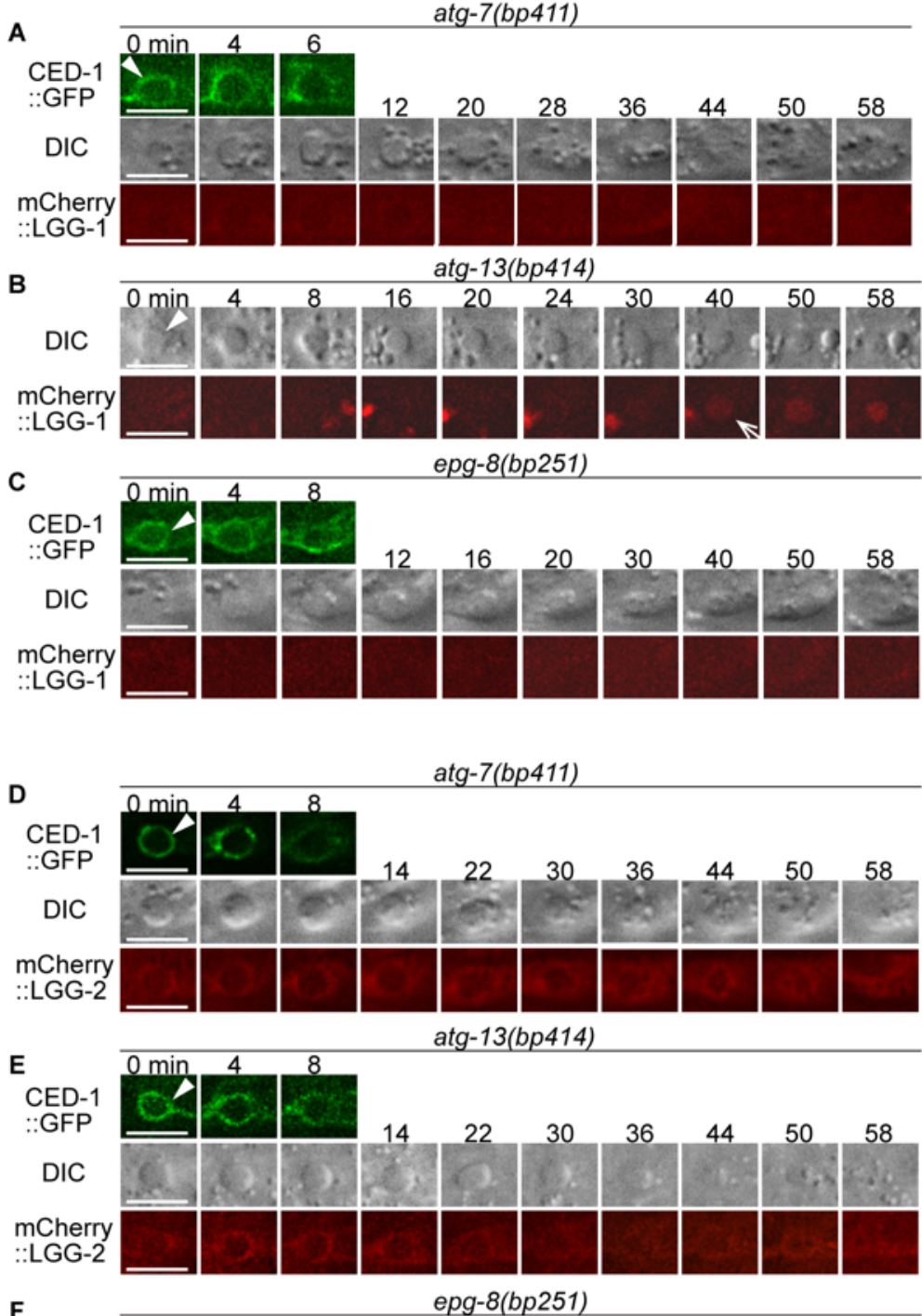

$\operatorname{atg}-7(b p 411)$

atg-13(bp414)

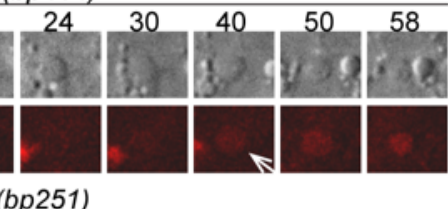

$\mathrm{F}$

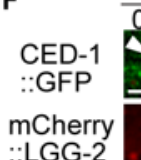

epg-8(bp251)

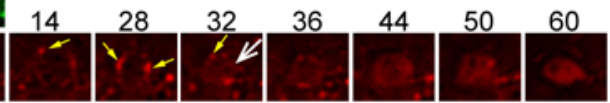
severely defective.
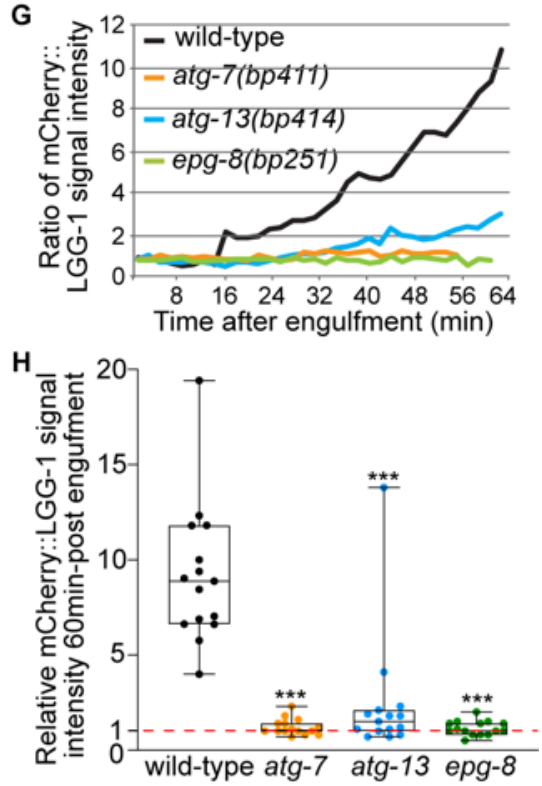

I

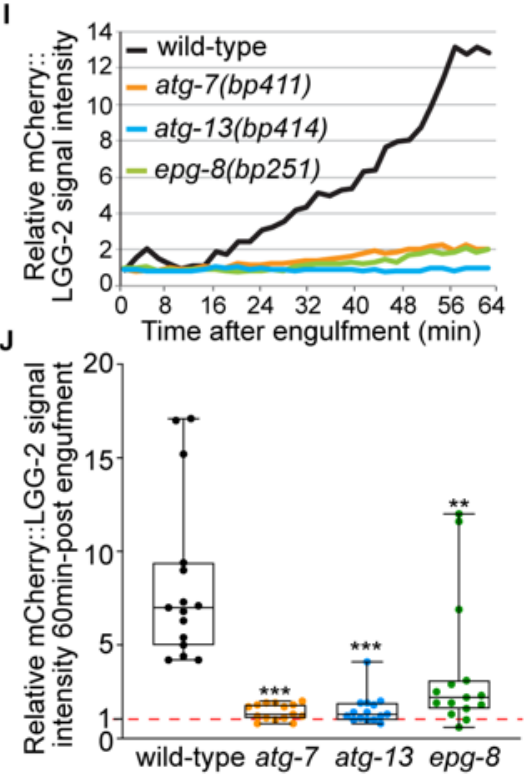

Figure 2. In autophagosome-formation mutants, the enrichment of the LGG vesicles on the phagosomal surface and the entry of the LGG signal into the phagosomal lumen are both 
246 (A-F) Time-lapse image series monitoring the enrichment of the puncta labeled with mCherry-tagged 247 LGG-1 (A-C) or LGG-2 (D-F) on phagosomes (white arrowheads) and the subsequent entry of mCherry 248 signal into the phagosomal lumen in atg-7, atg-13, and epg-8 mutant embryos. DIC images mark the 249 position of the cell corpse. "0 min" is when a phagosome is just sealed (determined by CED-1::GFP). "*” 250 denotes the time point that the mCherry signal starts to appear inside the phagosomal lumen. Scale 251 bars are $2 \mu \mathrm{m}$. Yellow arrows in $(F)$ mark mCherry::LGG-2 puncta on the surface of a phagosome 252 (open white arrow).

253 (G and I) The relative mCherry::LGG-1 or -2 signal intensity in the center of a phagosome (Y-axis) over 254 time (in 2-min interval) (X-axis). "0 min" indicates the moment when a phagosome is just sealed. (G) 255 The data for the wild-type, atg-7(bp411), atg-13(bp414), and epg-8(bp251) mutant embryos are from 256 Figures 1E and 2(A-C), respectively. (I) The data for the wild-type, atg-7(bp411), atg-13(bp414), and 257 epg-8(bp251) mutant embryos are from Figures 1 ll and 2(D-F), respectively.

$258(\mathrm{H}$ and $\mathrm{J})$ Box-and-Whiskers plots of the relative mCherry signal intensity measured in the center of 259 phagosomes 60 min-post the formation of nascent C3 phagosomes from 15 each of wild-type, atg260 7(bp411), atg-13(bp414), and epg-8(bp251) mutant embryos. Red dashed lines indicate the position of 261 value 1, which represents no signal enrichment relative to the background signal. ${ }^{* * *}, p<0.001,{ }^{* *},<0.001 p<0.01$, Student $t$-test of each mutant comparing to the wild-type value.

To further confirm that autophagosomes fuse to phagosomes, we examined the subcellular localization of a mCherry::ATG-9 reporter expressed in engulfing cells. Besides the LGG proteins, ATG-9 is also an established autophagosomes marker as it is the only transmembrane protein in autophagosomes [37]. In embryonic hypodermal cells that co-expressed mCherry::ATG-9 and either mNG::LGG-1 or::LGG-2, puncta labeled with mCherry are recruited to the surfaces of phagosomes (Fig 272 phagosomal lumen was nearly perfect (Fig 3). Together, the above observations verified that 273 autophagosomes on which membranes ATG-9 and LGG proteins are present are recruited to 274 phagosomal surfaces and subsequently fuse to phagosomes containing apoptotic cells. 


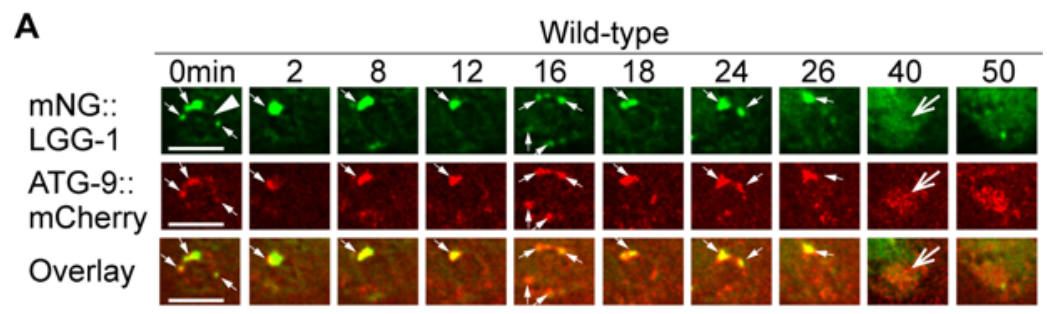

B

\section{Figure 3. LGG-1 and LGG-2 puncta colocalize with ATG-9, a component of autophagosomes.}

277 The $\mathrm{mNG}$ - and $\mathrm{mCherry-tagged} \mathrm{reporters} \mathrm{are} \mathrm{expressed} \mathrm{in} \mathrm{wild-type} \mathrm{embryos} \mathrm{under} \mathrm{the} \mathrm{control} \mathrm{of} \mathrm{P}_{\text {ced- }}$

278 1. White arrowheads make nascent phagosomes. Small white arrows mark the regions where LGG

279 and ATG $-9^{+}$puncta colocalize. Open white arrows indicate the moment when the fluorescent signal is

280 first detected inside the phagosomal lumen. Scale bars are $5 \mu \mathrm{m}$.

281 (A) Time-lapse microscopy showing the localization of mNG::LGG-1 and mCherry::ATG-9. Images are 282 from ABplaapppp, which engulfs C3.

283 (B) Time-lapse microscopy showing the localization of mNG::LGG-2 and mCherry::ATG-9. Images are 284 from ABplaapppa, which engulfs C1.

\section{Autophagosomes facilitate the degradation of apoptotic cells inside phagosomes}

To examine whether the incorporation of autophagosomes into phagosomes affects the clearance of the engulfed apoptotic cells, we first quantified whether, in mutants of genes essential for the biogenesis of autophagosomes, apoptotic cells were un-degraded and persistent in embryos. In addition to the atg-7, atg-9, atg-13, and epg-8 mutants characterized above, we also characterized loss-offunction mutants of $\operatorname{lgg}-1$ and $\operatorname{lgg}-2$, and of atg-3, whose gene product is essential for the conjugation of

295 post the first embryonic cell division, an average of 11.3 cell corpses were scored (Fig 4A). The mutant 296 2-fold embryos examined bore 46.9\% - 91.2\% more cell corpses (Fig 4), indicating that the clearance of 297 cell corpses is defective. Together, the findings reported in Figs 2, 3, and 4 indicate that 298 autophagosomes made a substantial contribution to the clearance of cell corpses. 
To determine whether the lack of autophagosomes impairs the engulfment or degradation of cell 300 corpses, we monitored the formation and degradation of phagosomes containing apoptotic cells $\mathrm{C} 1, \mathrm{C} 2$, 301 and C3 (Fig 1B) in wild-type, atg-7, and lgg mutant embryos in real-time using established protocol 302 (Materials and Methods) [24,27]. The CED-1::GFP expressed in engulfing cells labels the extending 303 pseudopods and enables us to monitor the process of phagosome formation, starting from the budding 304 and ending at the sealing of the pseudopods [20,21]. 2xFYVE::mRFP (also expressed under $\mathrm{P}_{c e d-1}$ ), a 305 reporter for phagosomal surface PtdIns(3)P, enables us to monitor the shrinking of a phagosome, an 306 indication of phagosome degradation [19,23,24]. In atg-7, lgg-1, and lgg-2 mutant strains that co307 expressed CED-1::GFP and 2xFYVE::mRFP, we found that engulfment was completed in 4-8 min, just 308 like in wild-type embryos (Fig 5 A-E), indicating that defects in autophagosomes biogenesis do not 309 affect the engulfment of cell corpses. However, the lifespans of phagosomes (Materials \& Methods) 310 were much longer in $\operatorname{atg}-7, \operatorname{lgg}-1$, and $\operatorname{lgg}-2$ mutants than in wild-type embryos (Fig 5 A-D, F). All 311 wild-type phagosomes have a lifespan between 40-60 min (Fig 5F). In atg-7, lgg-1, and lgg-2 mutants, 312 the lifespan varied in a much more extensive range. Remarkably, $43.8 \%, 53.3 \%$, and $82.4 \%$ of 313 phagosomes in atg-7, lgg-2, and lgg-1 mutant embryos, respectively, have lifespans longer than 60 min 314 (Fig 5F). The longest persisting phagosome, which was observed in an lgg-1 mutant embryo, lasted 315 longer than 136 min (Fig 5D). These observations indicate that autophagosomes made a specific contribution to the efficient degradation of phagosomal contents. 
A

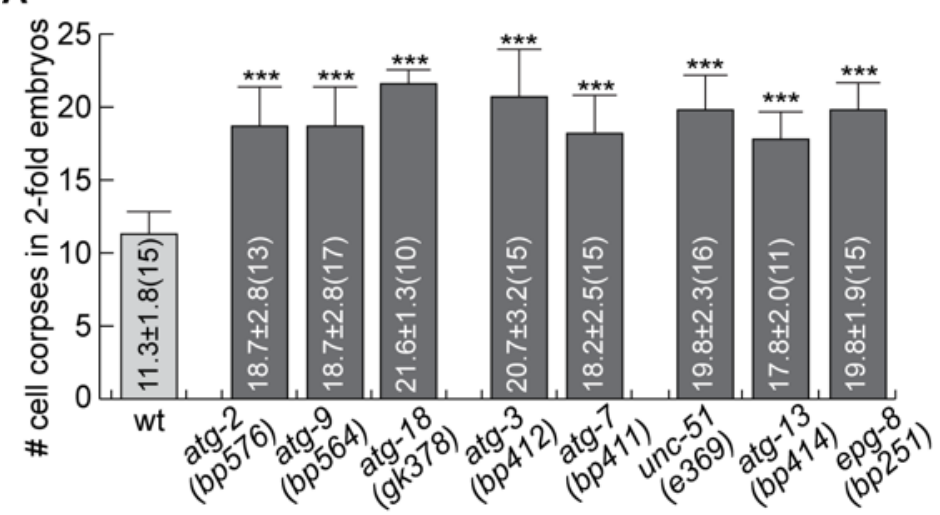

C
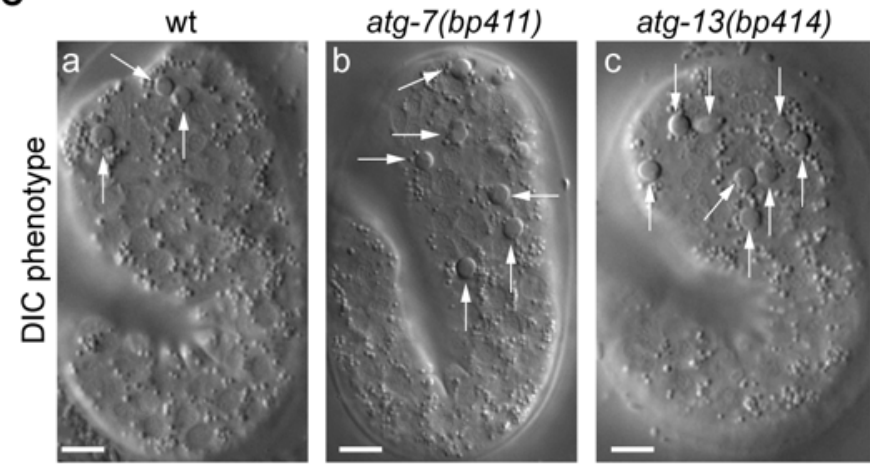

B

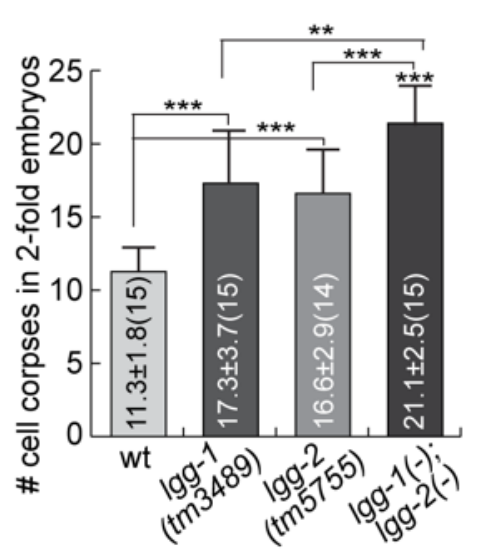

$\operatorname{lgg}-1$ (tm3489);

epg-8(bp251)

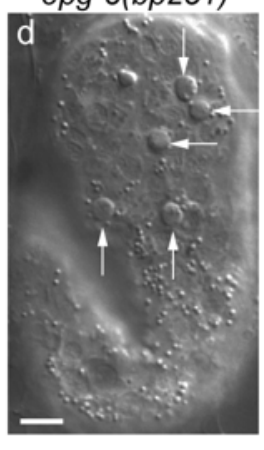

$\operatorname{lgg}-2(\operatorname{tm} 5755)$

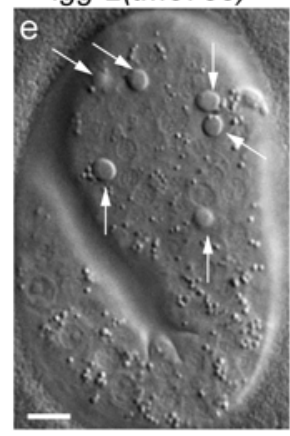

Figure 4. Mutations in autophagy genes impair the clearance of apoptotic cells.

319 (A-B) Bar graph displaying the average numbers of somatic cell corpses in 2-fold stage wild-type and

320 various mutant embryos. Bars and error bars represent mean and standard deviation (sd),

321 respectively, the actual values of which are displayed inside the bars. Numbers in parentheses indicate

322 the number of embryos scored. ${ }^{* * *}, p<0.001$, Student $t$-test of each mutant comparing to the wild-type 323 value.

324 (C) DIC images of cell corpses in 2-fold stage embryos of various genotypes. White arrows indicate 325 button-like structures characteristic of cell corpses. Scale bars are $5 \mu \mathrm{m}$. 


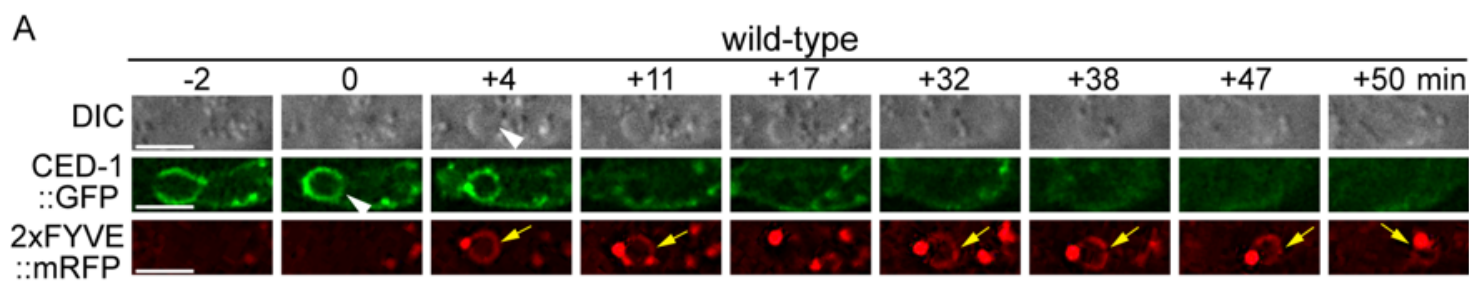

B

\begin{tabular}{|c|c|c|c|c|c|c|c|c|c|c|}
\hline & -2 & 0 & +4 & +12 & +16 & +24 & +34 & +46 & +60 & $+74 \mathrm{~min}$ \\
\hline DIC & & & 8 & 83 & Cos & G4 & & & & 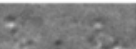 \\
\hline $\begin{array}{l}\text { CED-1 } \\
: \because G F P\end{array}$ & 2 & & & $->0$ & -2 & 4 & & & & 4 \\
\hline $\begin{array}{l}\text { XFYVE } \\
\text { mRFP }\end{array}$ & & & & & & & & & & 8 \\
\hline
\end{tabular}

C

$\operatorname{lgg}-2(\operatorname{tm} 5755)$

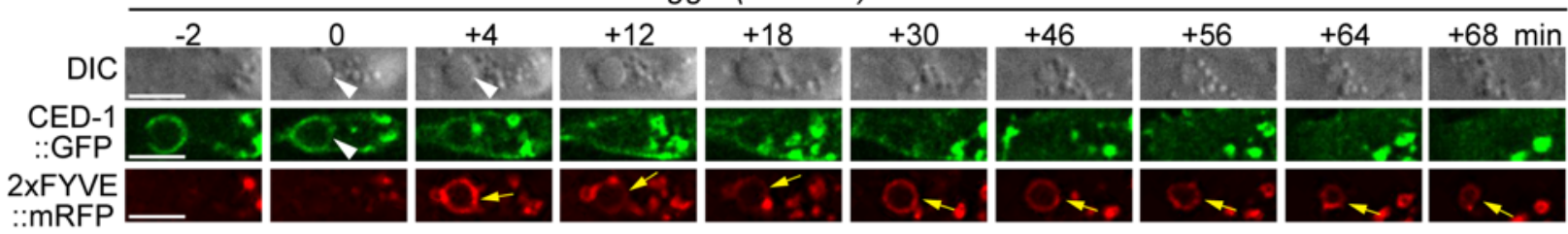
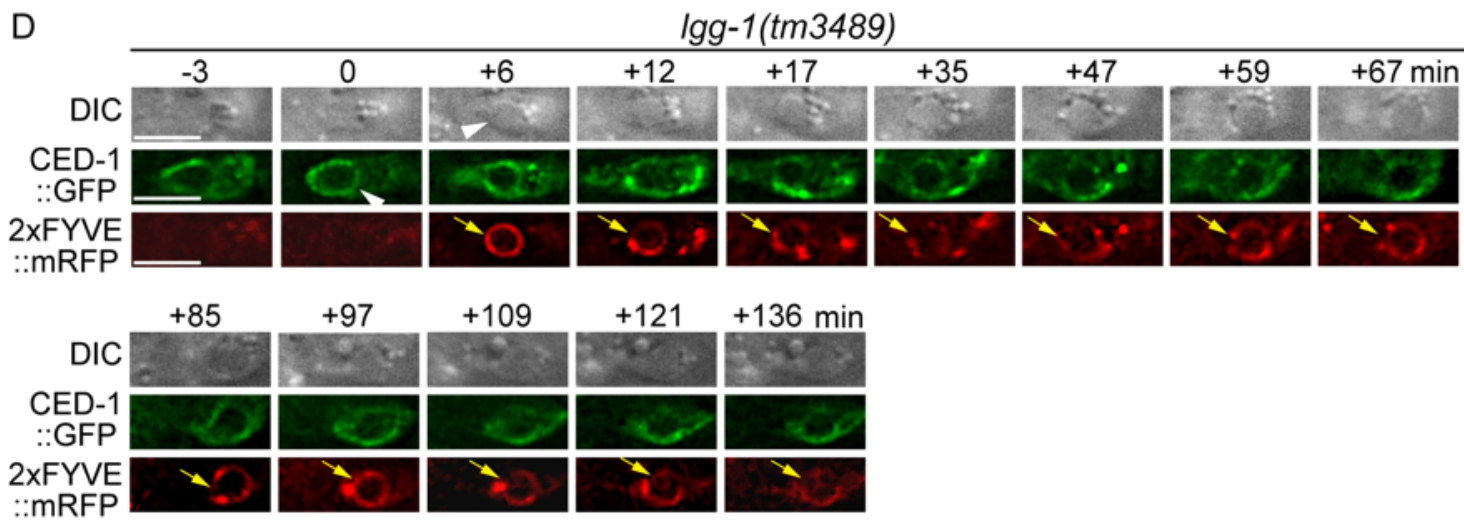

E

328 Figure 5. Mutations in atg-7, lgg-1, and lgg-2 delay the degradation of cell corpses.

329 (A-D) Time-lapse recording conducted in wild-type and different mutant embryos monitoring the

330 dynamics of the pseudopod marker CED-1::GFP and the phagosome marker 2xFYVE::mRFP (both

331 expressed in engulfing cells) during the engulfment and degradation processes of cell corpse C3 by

332 ABplaapppp. "0 min" is the first time point when a nascent phagosome (white arrowheads) is formed,

333 as indicated by the closure of a green GFP::CED-1 ring. 2xFYVE::mRFP labels the surface of a

334 phagosome (yellow arrows) until it is degraded. Scale bars are $2 \mu \mathrm{m}$. 
335 (E) Histogram depicting the distribution of the time it takes to engulf 15 C3 cell corpses in each of the

336 four genotypes. The engulfment time is defined as the period between the first time point when

337 pseudopods (labeled with CED-1::GFP) are spotted and the time point when a full circle forms around

338 C3.

339 (F) Histogram depicting the distribution of the duration of 15 C3 phagosomes in each of the four 340 genotypes. Phagosome lifespan is measured as the time interval between the " 0 min" time point and 341 the time point when the phagosome shrinks to one-half of its original diameter.

LGG-1 and LGG-2 act in engulfing cells, and together they define three subpopulations of autophagosomes that are incorporated into phagosomes

LGG-1 and LGG-2 have distinct structural features and were observed to attach to different subpopulations of autophagosomes, which represent autophagosomes at different maturation stages [26,28].

The sub-populations of autophagosomes labeled by LGG-1- or LGG-2- are incorporated into phagosomes (Fig 1). lgg-1 and lgg-2 single mutants are both inefficient in clearing cell corpses (Fig 4B). Furthermore, in $\operatorname{lgg}-1$; $\operatorname{lgg}-2$ double mutant embryos at 2-fold, the Ced phenotype is further enhanced significantly (Fig 4B) -- the number of cell corpses is $22.0 \%$ and $27.1 \%$ larger than in the $\operatorname{lgg}$ 1 and lgg-2 single mutants, respectively. This enhanced phenotype indicates an additive effect and suggests that the autophagosomes labeled with LGG-1 or LGG-2 play parallel and partially redundant roles in promoting phagosome degradation. In embryos co-expressing GFP::LGG-1 and mCherry::LGG-2, we observed that on the surfaces of phagosomes, those puncta were either labeled with GFP mCherry alone or with both GFP and mCherry (Fig 6A). In hypodermal cells, puncta that were either labeled with GFP mCherry alone or with both GFP and mCherry but were not attached to phagosomes were also observed (Fig 6B). These observations indicate that in addition to the LGG- $1^{+}-$ only and LGG-2+-only subpopulations, a third LGG-1 $1^{+} \mathrm{LGG}-2^{+}$double-positive subpopulation of autophagosomes exists. This subpopulation likely corresponds to an intermediate stage during the maturation path of autophagosomes.

366 tagged corresponding $\lg g$ cDNA efficiently rescued the Ced phenotype (Fig S5). The $g f p:: \operatorname{lgg}-1$ and $367 m$ Cherry::lgg-1 transgenes lowered the number of cell corpses from on-average 152\% of wild-type level 
368 observed in the $\operatorname{lgg}-1(\operatorname{tm} 3489)$ mutants to $118 \%$ and $123 \%$ of the wild-type level, respectively.

369 Similarly, the $g f p:: \operatorname{lgg}-2$ and $m$ Cherry::lgg-2 transgenes lowered the number of cell corpses from on-

370 average $143 \%$ of wild-type level observed in the $\operatorname{lgg}-2(\operatorname{tm} 5755)$ mutants to $109 \%$ and $112 \%$ of the wild371 type level, respectively (Fig S5). These results indicate that $l g g-1$ and $l g g-2$ primarily act in engulfing 372 cells to facilitate the clearance of apoptotic cells.

A

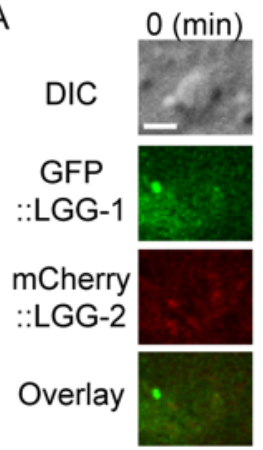

B

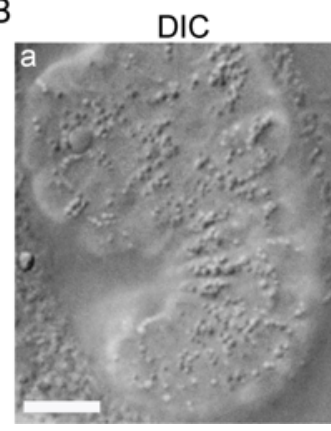

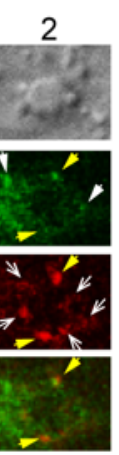

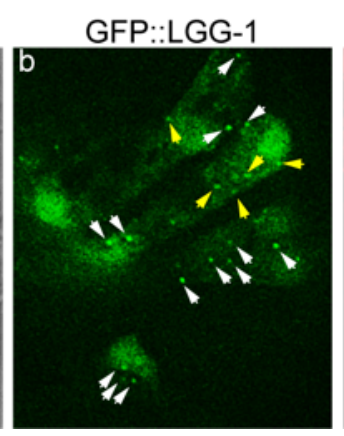

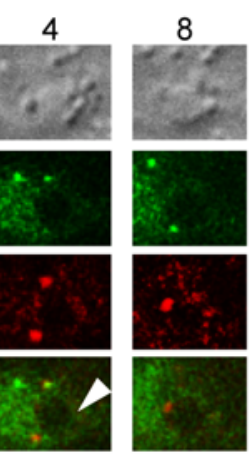

GFP::LGG-1
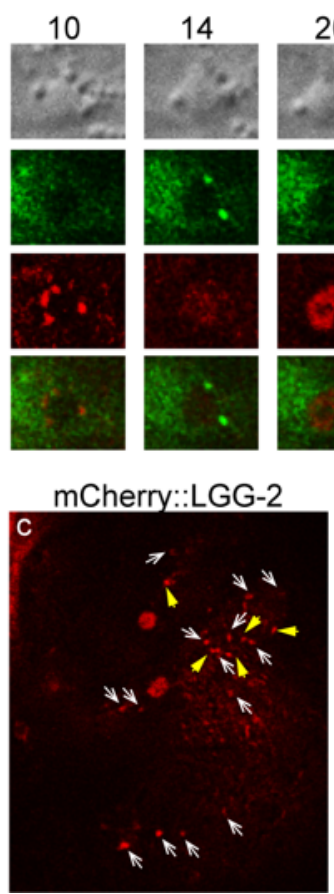
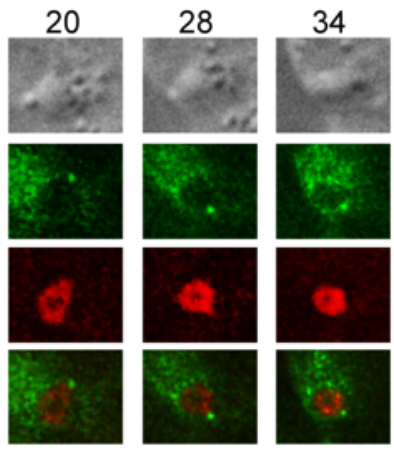

Overlay

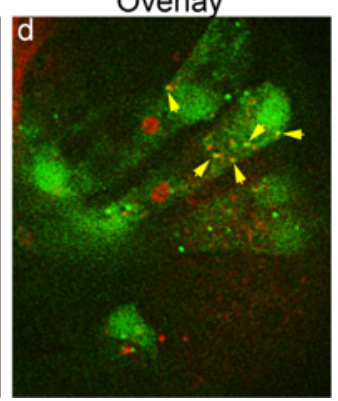

Figure 6. The puncta labeled with LGG-1 and/or LGG-2 define three distinct populations of vesicles

The time-lapse recording was conducted on wild-type embryos co-expressing $\mathrm{P}_{\text {ced-1 }} g f p::$ Igg-1 and $\mathrm{P}_{\text {ced- }}$ ${ }_{1} m$ Cherry::Igg-2.

(A) Time-lapse image series of a C2 phagosome (one big white arrowhead in the " 4 min" image). " 0 min" is the moment when a nascent phagosome just seals. The scale bar is $2 \mu \mathrm{m}$. The white arrows, white open arrows, and yellow arrows in the "2 min" image panels mark GFP' mCherry", GFP' $\mathrm{mCherry}^{+}$, and $\mathrm{GFP}^{+} \mathrm{mCherry}^{+}$puncta on the surface of the phagosome, respectively.

(B) DIC and fluorescence images of an embryo exhibiting $\mathrm{LGG}^{+}$puncta outside phagosomes in multiple cells. The scale in (a) bar is $10 \mu \mathrm{m}$. The white arrows, white open arrows, and yellow arrows mark $\mathrm{GFP}^{+}$mCherry, GFP- $\mathrm{mCherry}^{+}$, and $\mathrm{GFP}^{+} \mathrm{mCherry}^{+}$puncta on the surface of the phagosome, respectively. 
The small GTPase RAB-7 is recruited to the phagosomal membrane shortly after the formation of a C. elegans phagosome [19]. RAB-7 is also enriched on the surface of lysosomes [19]. Mammalian

391 RAB7 and C. elegans RAB-7 mediate the fusion between the maturing phagosome and lysosomes and do that through their effector, the HOPS complex [3,25,39]. In addition, mammalian RAB7 is known to drive lysosome-autophagosome fusion through the HOPS complex and PLEKHM1, an adaptor protein [40,41]. C. elegans RAB-7 also plays an essential role in autolysosome formation [26], although whether it is localized to autophagosomes was not reported previously. To examine whether RAB-7 is localized to $\mathrm{LGG}-1^{+}$and LGG-2 ${ }^{+}$autophagosomes that fuse to C. elegans phagosomes, we generated two transgenic $C$. elegans strains that co-expressed the mCherry::LGG-1/ GFP::RAB-7 or mCherry::LGG-2/ GFP::RAB-7 pairs of reporters (Materials and Methods). We observed that GFP::RAB-7 was localized to some but not all of the LGG-1 ${ }^{+}$or $\mathrm{LGG}-2^{+}$puncta (Fig 7 B-D). The $\mathrm{LGG}^{+} \mathrm{RAB}-7^{+}$double-positive autophagosomes were observed both freely distributed in the cytoplasm of ventral hypodermal cells (Fig 7B(a-c) and $\mathbf{C}$ ) and on phagosomal surfaces (Fig $7 \mathbf{B}(\mathbf{d}-\mathbf{f})$, D). In addition, Fig 7B(d-f) depicts that GFP::RAB-7 is both evenly distributed to the surface of a phagosome (d) as previously reported [19], and highly enriched on LGG-1 ${ }^{+}$autophagosomes that are recruited to the phagosomal surface (d-f, white arrows). Fig 7D shows a dynamic fusion event of an LGG-2 ${ }^{+} / \mathrm{RAB}-$ $7^{+}$punctum (marked by the bottom arrow in the " 0 min" time point) attached to the phagosome membrane and becoming part of the phagosomal surface at the " +6 min" time point. On the other hand, we also observed puncta that were $\mathrm{LGG}^{+}$but RAB-7- (Fig $7 \mathrm{~B}$ and $\mathrm{C}$, white arrowheads), which were likely autophagosomes not acquired RAB-7 yet, and puncta that were $\mathrm{LGG}^{-}$but RAB-7 ${ }^{+}$(Fig 7 B and C, yellow arrows), which represented intracellular organelles such as late endosomes or lysosomes. In conclusion, RAB-7 is localized to the majority but not all autophagosomes; furthermore, all autophagosomes that were observed on phagosomal surfaces were RAB- $7^{+}$.

We next examined whether the recruitment and fusion of LGG-1 ${ }^{+}$- or LGG-2 ${ }^{+}$- autophagosomes to the $\mathrm{C} 1, \mathrm{C} 2$, and $\mathrm{C} 3$ phagosomes were normal in rab-7(ok511) null mutant embryos [19]. The enrichment of mCherry ${ }^{+}$puncta on the surfaces of phagosomes indicates the recruitment event, whereas the accumulation of the mCherry signal inside the phagosomal lumen indicates the fusion event. In $r a b$ 7(ok511) mutants, robust enrichment of both the mCherry::LGG-1 ${ }^{+}$and ::LGG-2 $2^{+}$puncta to phagosomal surfaces are prominent (Fig 7 E-F), indicating that the recruitment of autophagosomes was normal. However, no mCherry signal was observed entering the phagosomal lumen (Fig 7 E-F, Movies S4, S5). We quantified the time between the moments a phagosome was just born and that when an obvious mCherry signal was observed inside the phagosomal lumen. In the wild-type embryos, this time is $<30$ 
$421 \mathrm{~min}$ in $100 \%$ of the samples analyzed, and the median value is 14 and $18 \mathrm{~min}$ for LGG- $1^{+}$and LGG-2 $2^{+}$ 422 autophagosomes, respectively (Figs 1(E and $\mathbf{H})$ and 7(G-H)). In stark contrast, in rab-7 mutants, the 423 mCherry signal was not detected in the lumen inside any of the phagosomes for LGG-1 or LGG-2 424 reporters ( $\mathrm{n}=9$ for each reporter) over a time span of 0-60 min after phagosome formation (Fig 7 G-H). 425 For over half of the samples, the observation period was extended beyond 90 min after phagosome 426 formation, and still, no mCherry signal was observed in the phagosomal lumen within this period. 427 These results strongly indicate that RAB-7 function is absolutely needed for 428 autophagosomes/phagosome fusion but not required for the recruitment of autophagosomes to 429 phagosomal surfaces.

430 In C. elegans, a null mutation in vps-18, which encodes VPS-18, a subunit of the HOPS 431 complex, impairs phagosome maturation [42]. The lack of autophagosome-phagosome fusion observed 432 in rab-7 mutants hinted us to subsequently examine the vps-18 null mutant embryos. We found that, 433 like in $r a b-7$ mutants, the recruitment of autophagosomes to phagosomes appeared normal in $v p s-18$ 434 mutants (Fig 7 E-F). Also, like in rab-7 mutants, in vps-18 mutants, the fusion of LGG-1 ${ }^{+}$and LGG-2 $2^{+}$ 435 autophagosomes to phagosomes was severely defective (Fig 7 E-H). However, whereas in $r a b-7$ 436 mutants, the entry of mCherry signal to phagosomal lumen was blocked completely in all samples, in 437 some vps-18 mutant embryos, the entry of mCherry signal still occurred, albeit severely delayed. In 438 contrast, in other samples, the entry was blocked (Fig $7 \mathbf{E - H})$, indicating a fusion defect that is less 439 severe than that caused by the rab-7 null mutation and suggesting the existence of the residue HOPS 440 function in vps-18 mutants. Together, our above observations indicate that RAB-7 and the HOPS 441 complex play a critical and specific role in the fusion between autophagosomes and phagosomes. 

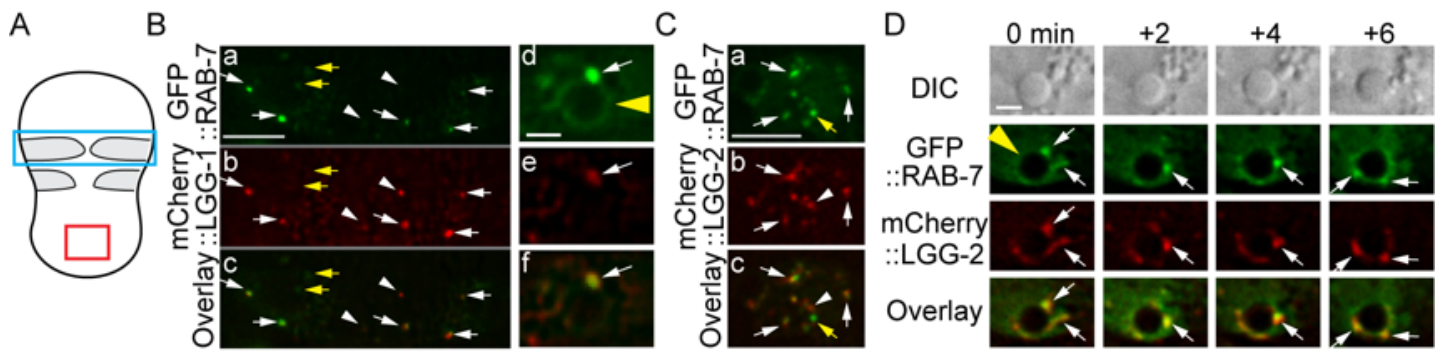

$\mathrm{E}$

mCherry::LGG-1
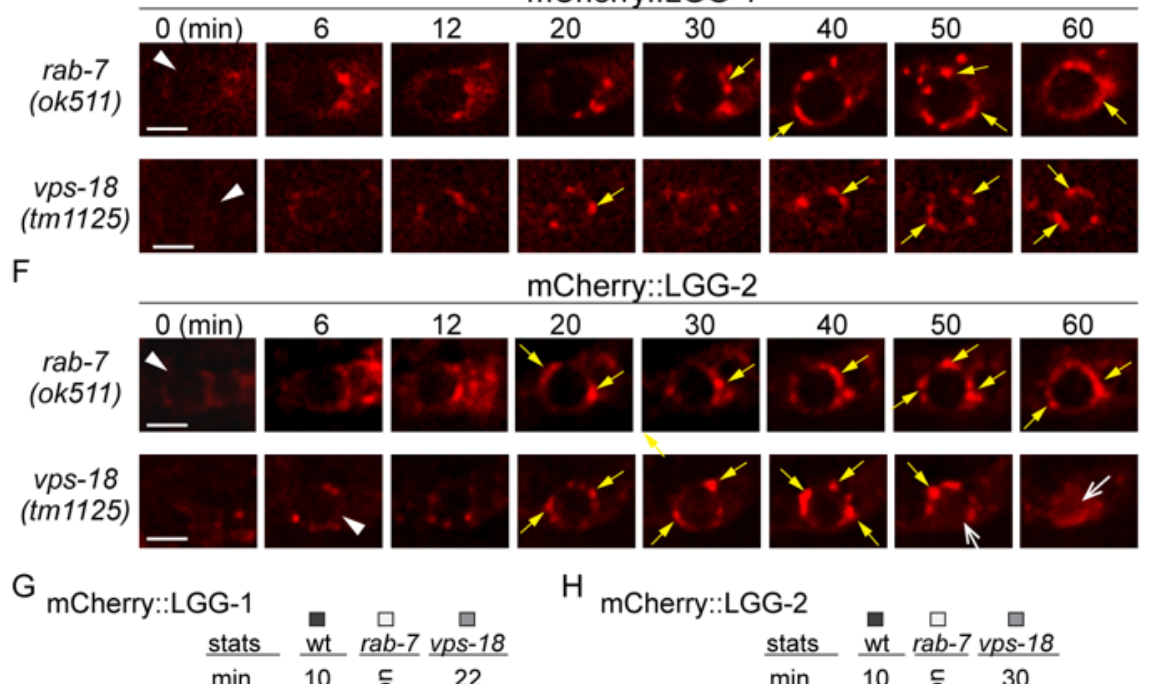

H mCherry::LGG-2
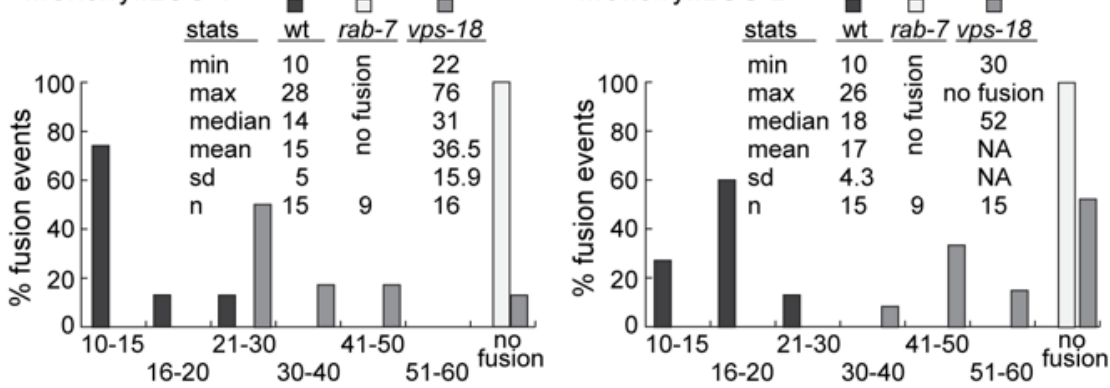

The moment ( $\mathrm{min})$ when fusion starts

The moment $(\mathrm{min})$ when fusion starts

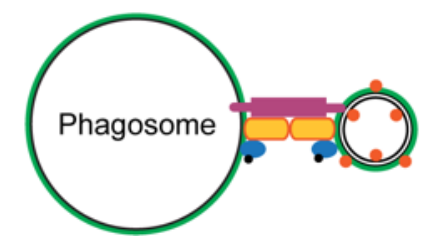

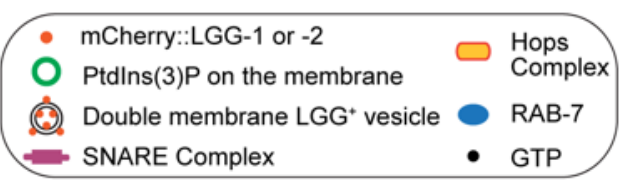

Figure 7. RAB-7 and VPS-18 are essential for the fusion between autophagosomes and phagosomes.

446 (A) Diagram of the ventral surface of an embryo at $\sim 330$ min post $1^{\text {st }}$ embryonic division.

447 (B-D) Images of part of the ventral surface of an embryo co-expressing $\mathrm{P}_{\text {ced-1 }}$ gfp::rab-7 and $\mathrm{P}_{\text {ced- }}$

448 1mCherry::Igg-1 (B) or $\mathrm{P}_{\text {ced-1 }}$ mCherry::Igg-2 (C-D). B(a-c) depicts the region framed by the blue box in 449 (A). B(d-f) depicts a $\mathrm{C} 1$ phagosome (a yellow arrowhead). $\mathrm{C}(\mathrm{a}-\mathrm{c})$ depicts the region framed by the red 450 box in (A). (D) A time-lapse image series of a $C 1$ phagosome (a yellow arrowhead) indicates the 451 dynamic recruitment and fusion of GFP and mCherry double-positive puncta to the phagosomal 452 membrane. " 0 min" is the moment when the first puncta are observed on the phagosomal surface. 453 White arrows mark several puncta that are both $\mathrm{GFP}^{+}$and $\mathrm{mCherry}{ }^{+}$. Yellow arrows mark puncta that 
454 are GFP ${ }^{+}$but mCherry". White arrowheads mark puncta that are GFP- but mCherry ${ }^{+}$. Scale bars for $455 B(a-c)$ and $(C)$ are $5 \mu m$, and for $B(d-f)$ and $(D)$ are $2 \mu \mathrm{m}$.

456 (E-F) Time-lapse images monitoring the recruitment and fusion of puncta labeled with mCherry::LGG-1 457 (E) or::LGG-2 (F) to the C1, C2, and C3 phagosomes in rab-7(ok511) and vps-18(tm1125) mutant 458 embryos. "0 min" represents the moment when a phagosome just seals (white arrowheads). Yellow 459 arrows mark the $\mathrm{mCherry}^{+}$puncta on the phagosomal surfaces. One open white arrow marks the 460 mCherry signal inside the phagosomal lumen. Scale bars are $2 \mu \mathrm{m}$.

461 (G-H) Histograms depicting the distribution of the time it takes for LGG-1 ${ }^{+}$or LGG-2 ${ }^{+}$puncta to fuse to 462 phagosomes, measured from the " 0 min" point to the time point when mCherry was detected in the 463 center of a phagosome. C1, C2, and C3 phagosomes were recorded. $n$, the number of phagosomes 464 scored. "No fusion": no mCherry signal entry was observed even after 72-114 min post-nascent 465 phagosome formation. NA: not applicable.

466 (I) A Diagram depicting the mechanism driving autophagosome-phagosome fusion. RAB-7 is enriched 467 on the surfaces of both phagosomes and autophagosomes. RAB-7 and VPS-18, a subunit of the HOPs complex, are proven essential for autophagosome-phagosome fusion. Other factors are proposed to play roles in this event based on the knowledge of intracellular membrane fusion in general.

\section{The CED-1 pathway drives the recruitment of autophagosomes to phagosomes}

The signaling pathway led by the phagocytic receptor CED-1 plays essential roles in initiating the maturation of phagosomes containing apoptotic cells [19]. The CED-1 pathway is known to drive the incorporation of early endosomes and lysosomes to phagosomes [19,21]. Here we further examined whether the loss-of-function mutations in members of the CED-1 pathway affect the incorporation of autophagosomes to phagosomes. In ced-1(e1735) null mutant embryos [20] that express the mNG::LGG-1 or -2 reporters, we observed severe defects in the incorporation of autophagosomes into phagosomes. First of all, only a very dim mNG signal was observed inside the phagosomal lumen 50 min post phagosome formation, a time point well past the observed initiation time for autophagosomes/phagosome fusion in the wild-type condition (Fig 8 A, C, F, and G, Movie S6). Whereas in wild-type embryos, the median relative LGG-1 and -2 signal intensities are 6.3 and 5.3 at 50 min-post phagosome formation, respectively, in ced-1 mutant embryos, the median values are merely 2.1 and 1.6, respectively (Fig 8 K-L). Secondly, unlike in rab-7 mutants, where autophagosomes were observed accumulating on phagosomal surfaces (Fig 7), in ced-1 mutants, very few LGG-1- or LGG-2labeled puncta were observed on phagosomal surfaces (Fig 8 A, C).

Further quantitative measurement of the samples presented in (Fig 8 A, C) and 14 additional samples for each of the reporters confirmed that the mNG signal was not enriched on the surfaces of 
488 phagosomes, in contrast to wild-type embryos (Fig 8 H-L). These results indicate a severe defect in the recruitment of autophagosomes to phagosomes. Unlike in the atg mutants that we have examined (Figs S2 and S3), in ced-1 mutant embryos, normal numbers of LGG-1 ${ }^{+}$or LGG-2 $2^{+}$puncta were observed (Fig S6), indicating that the biogenesis of autophagosomes is normal. Thus the recruitment defect observed in ced-1 mutants is not a consequence of the lack of autophagosomes; rather, it is likely a result of a defect in signaling between phagosomes and autophagosomes.

We further examined whether CED-6 and DYN-1, two other members of the CED-1 pathway, were also needed for the incorporation of autophagosomes into phagosomes. In the ced-6(n2095) and dyn-1(n4039) mutant embryos, the median relative LGG-1 and LGG-2 signal intensities in the center of phagosomes are much lower than that in wild-type samples, respectively, at 50 min-post phagosome formation mutants (Figs 8(B, D, F, G, K-L) and 9(A, D E, F, I, J)), although the defects are not as severe as in ced-1 mutants (Fig 8(A, C, F, G, K, L)). Further observation discovered that the LGG-1 or LGG-2-labeled puncta were rarely observed on the surfaces of phagosomes in these mutants (Figs 8(B,

501 D, I, J) and $\mathbf{9}(\mathbf{A}, \mathbf{F})$ ), demonstrating severe defects in the recruitment of autophagosomes to phagosomal 502 surfaces.

In ced-1, ced-6, and $d y n-1$ mutants, the fusion between autophagosomes and phagosomes might also be defective. However, the severe recruitment defects resulted in the lack of LGG-labeled puncta on phagosomal surfaces, making it difficult to evaluate whether there are additional fusion defects and how severe the fusion defects are.

We also examined whether the pathway composed of the small GTPase CED-10 and its bipartite Guanine Nucleotide Exchange Factor (GEF) CED-5 and CED-12, which acts parallel to the CED-1 pathway in the engulfment of cell corpses, plays any role in promoting the incorporation of autophagosomes into phagosomes. In the ced-5(n1812) null mutant and ced-10(n1993) loss-of-function mutant embryos, the accumulation of the LGG-1 and LGG-2 signals on the surfaces of the phagosomes and the subsequent accumulation of signals inside phagosomal lumen were normal both in the time course and in the levels of signal enrichment (Fig 9 B, C-E, G, H-J), indicating that both the recruitment and fusion of autophagosomes to phagosomes are normal. We thus conclude that, unlike the CED-1/-6/DYN-1 pathway, the CED-5/-10 pathway is not involved in regulating the incorporation of autophagosomes to phagosomes (Fig 9K). 
A

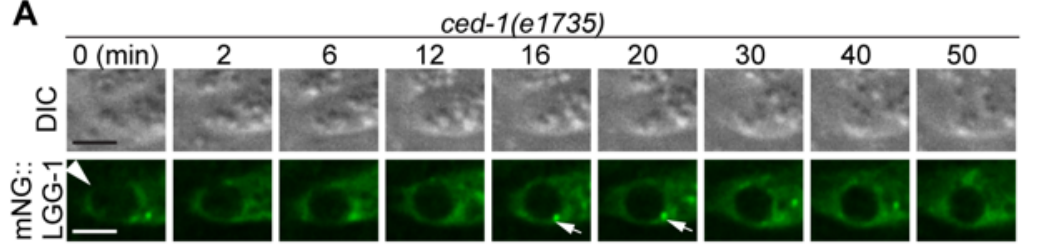

B

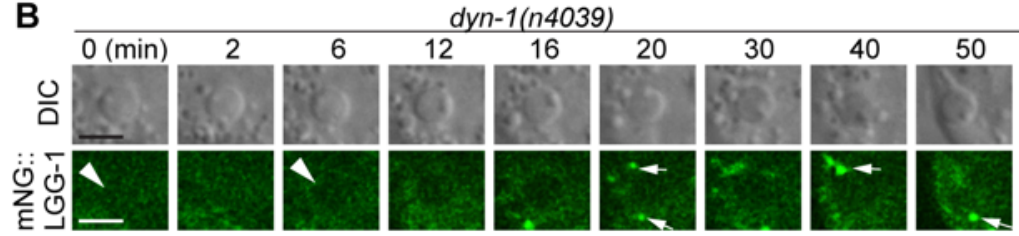

C

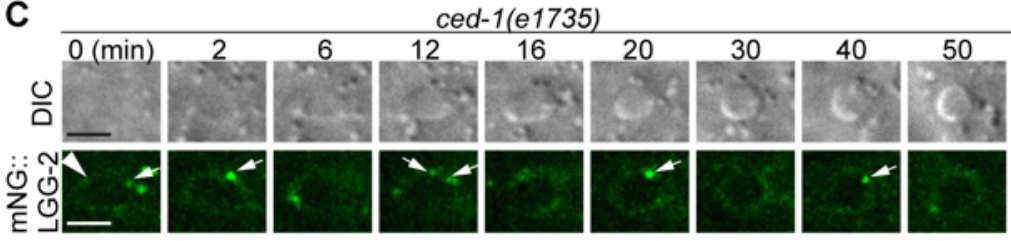

D

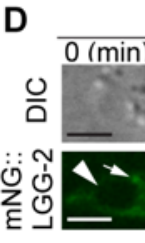

K

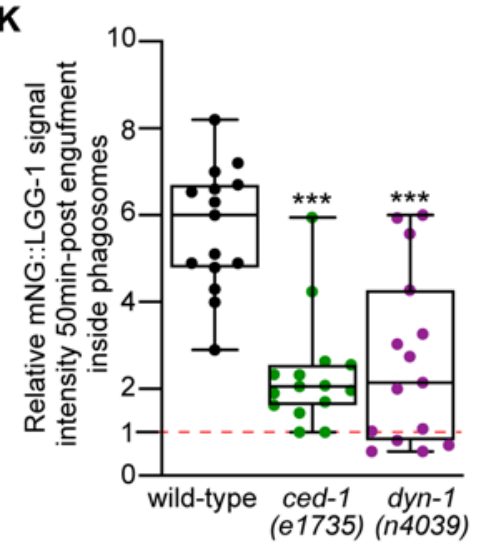

dyn-1(n4039)

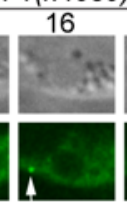

L

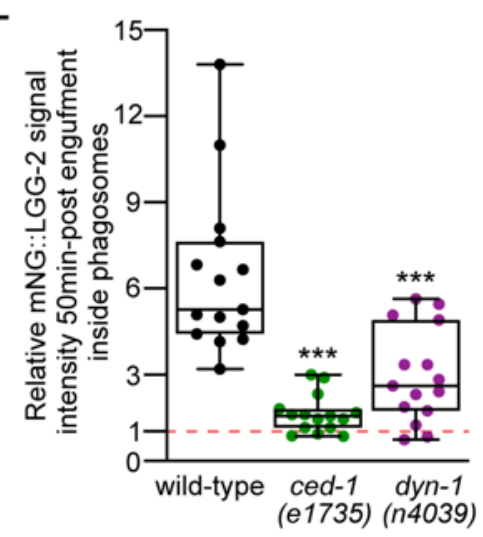

E
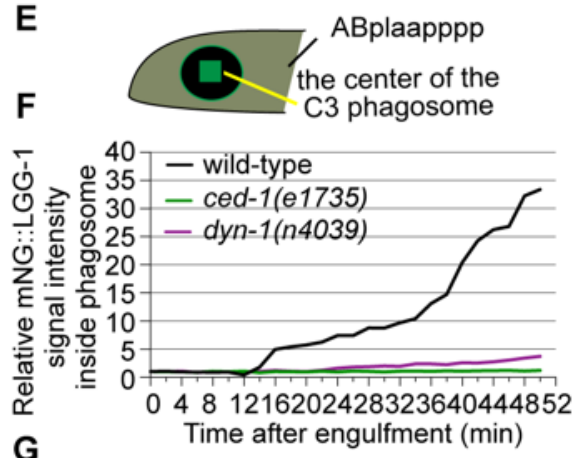

$\mathbf{G}$

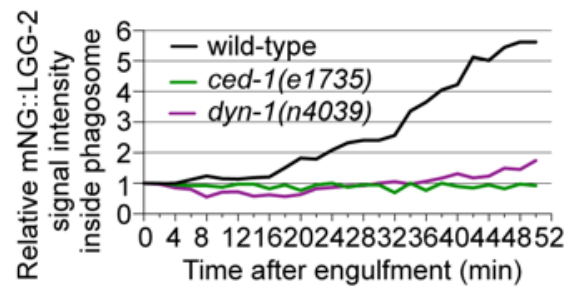

H

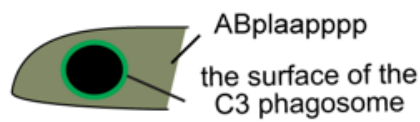

I

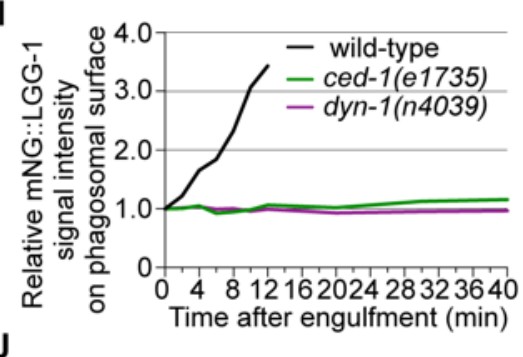

J

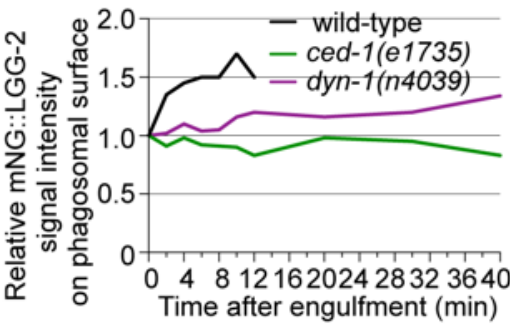

Figure 8. CED-1 and DYN-1 are essential for the incorporation of autophagosomes into

\section{9 phagosomes}

520 (A-D) Time-lapse image series monitoring the presence or absence of puncta (white arrows) labeled

521 with mNG::LGG-1 (A-B) or -2 (C-D) on C3 phagosomes (white arrowheads) and the subsequent entry

522 of the mNG signal into the lumen in ced-1 and dyn-1 mutant embryos. DIC images mark the position of

523 the cell corpse. "0 min" is the moment when phagosomes are just sealed. Scale bars are $2 \mu \mathrm{m}$.

524 (E) A diagram illustrating that the relative $\mathrm{mNG}$ signal in the center of a phagosome is measured over

525 time to create sub-figures $(F)$ and $(G)$. At time point t (time after "0 min"), the Relative Signal Intensity $T$

$526=\left(\right.$ Unit Intensity $(\text { phagosome center })_{\mathrm{T}}-$ Unit Intensity $\left.(\text { background })_{\mathrm{T}}\right) /($ Unit Intensity $($ phagosome

527 center) $)_{\mathrm{TO}^{-}}$Unit Intensity (background) $)_{\mathrm{TO}}$ ). 
528 (F-G) The relative mNG::LGG-1 (F) or -2 (G) signal intensity in the center of a phagosome (Y-axis) over 529 time in the 2-min interval (X-axis). "0 min" is the moment when pseudopods are sealed and a nascent 530 phagosome forms. (F) The data for the wild-type, ced-1(e1735), and dyn-1(n4039) mutant embryos are 531 from Figs $1 \mathrm{~F}$ and 8(A-B), respectively. (G) The data for the wild-type, ced-1(e1735), and dyn-1(n4039) 532 mutant embryos are from Figures 11 and 8(C-D), respectively.

$533(\mathrm{H})$ A diagram illustrating that the relative $\mathrm{mNG}$ signal on the surface of a phagosome is measured over 534 time to create sub-figures $(\mathrm{I})$ and $(\mathrm{J})$. At time point $\mathrm{T}$ (time after " $\mathrm{O}$ " min), the Relative signal intensity $535=(\text { Unit Intensity }(\text { phagosome surface } \text { (the green ring }))_{\mathrm{T}}-$ Unit Intensity $\left.(\text { background })_{\mathrm{T}}\right) /($ Unit 536 Intensity(phagosome surface) $)_{\text {TO }}$ - Unit Intensity (background)

537 (I-J) The relative mNG::LGG-1 or -2 signal intensity on the surface of a phagosome (Y-axis) over time 538 in the 2-min interval (X-axis). "0 min" indicates the moment when pseudopods are sealed and nascent 539 phagosome forms. (I) The data for the wild-type, ced-1(e1735), and dyn-1(n4039) mutant embryos are 540 from Figures 1F and 8(A-B), respectively. (J) The data for the wild-type, ced-1(e1735), and dyn541 1(n4039) mutant embryos are from Figs $1 \mathrm{l}$ and 8(C-D), respectively.

542 (K-L) Box-and-Whiskers plots of the relative $\mathrm{mNG}$ signal intensity measured in the center of 543 phagosomes 50 min-post the formation of nascent C3 phagosomes from 15 each of wild-type, ced544 1(e1735), and dyn-1(n4039) mutant embryos. Red dashed lines indicate the position of value 1, which 545 represents no signal enrichment relative to the background signal. ${ }^{* * *}, p<0.001$, Student $t$-test of each 546 mutant compared to the wild-type value. 
A mCherry::LGG-1

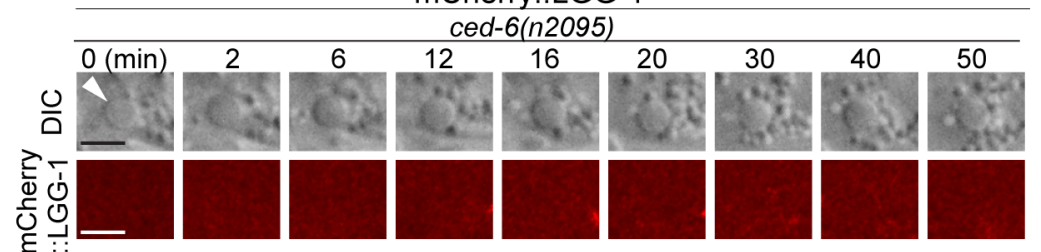

B \begin{tabular}{c}
\multicolumn{7}{c}{$c e d-5(n 1812)$} \\
\cline { 2 - 7 }
\end{tabular}

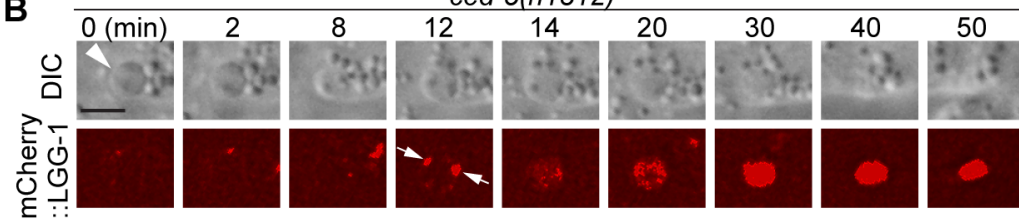

C

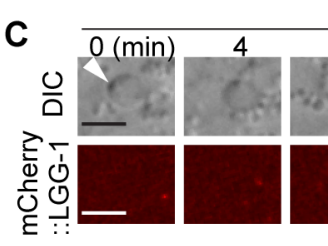

$\mathbf{F}$
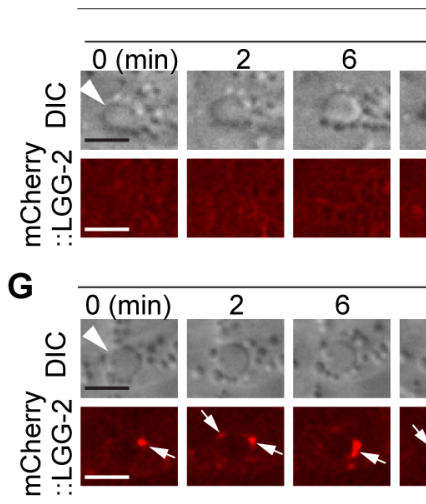

ced-10(n1993)
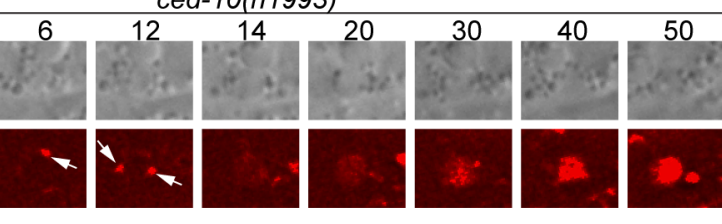

mCherry::LGG-2
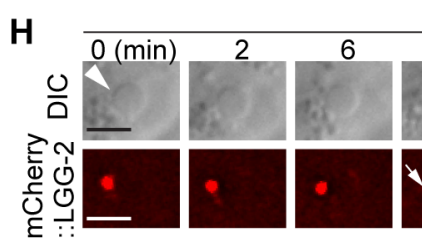

$\mathbf{K}$

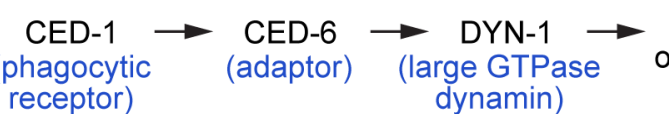

Recruitment

ced-10(n1993)

ced-5(n1812)
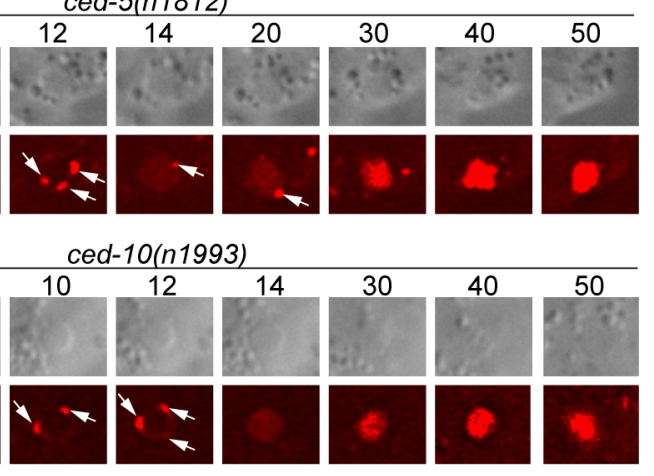
receptor) Not involved in the incorporation of autophagosomes
to phagosomes of autophagosomes to phagosomes
(SH2-containing adaptor) into phagosomes
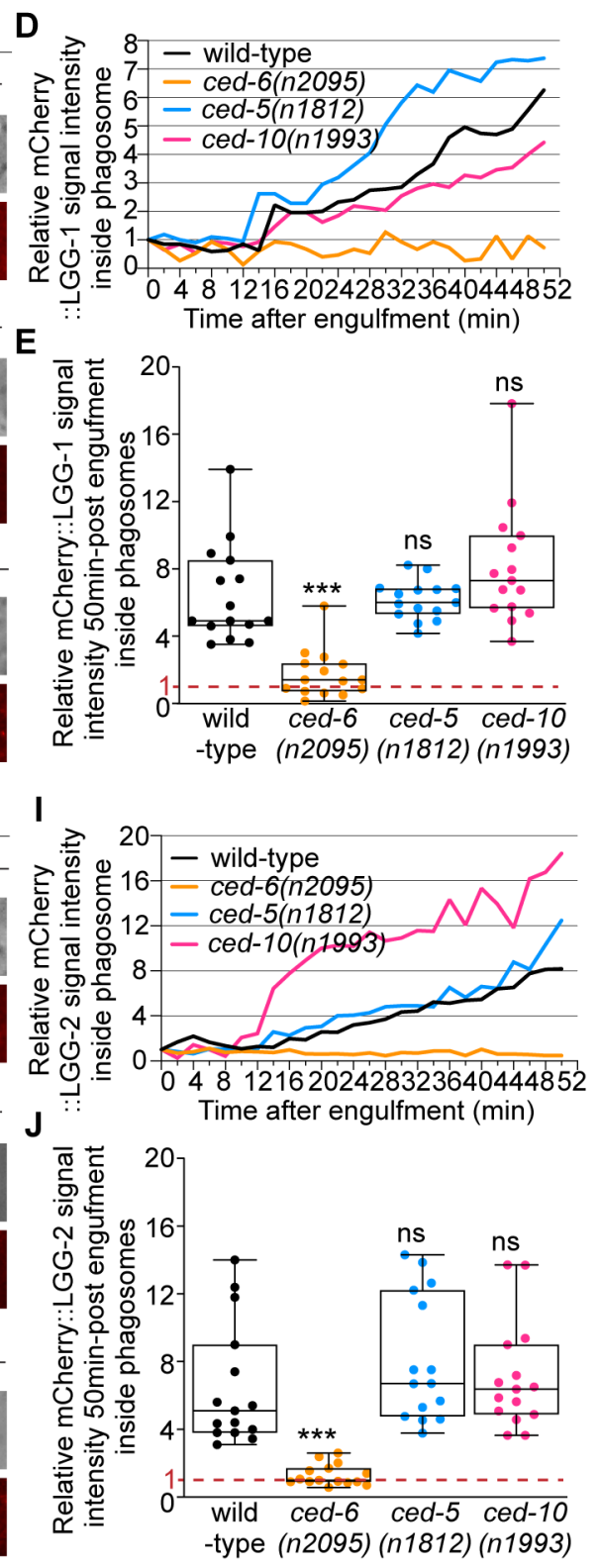

RAB-7

Fusion of (small GTPase) $\rightarrow$ autophagosomes The HOPS complex to phagosomes (RAB-7 effector)

Figure 9. ced-6, but not ced-5 or ced-10, is required for the incorporation of autophagosomes

(A-C and F-H) Time-lapse image series monitoring the presence or absence of puncta (white arrows) labeled with mCherry::LGG-1 (A-C) or -2 (F-H) on C3 phagosomes (white arrowheads) and the subsequent entry of the mCherry signal into the phagosomal lumen in ced-6, ced-5, and ced-10 mutant 
554 embryos. DIC images mark the position of the cell corpse. " $0 \mathrm{~min}$ " is the moment when a nascent 555 phagosome just seals. Scale bars are $2 \mu \mathrm{m}$.

556 (D and I) The relative mCherry::LGG-1 (D) or -2 (I) signal intensity in the center of a phagosome (Y557 axis) over time (in the 2-min interval) (X-axis). "0 min" indicates the moment when a nascent 558 phagosome just seals. (D) The data for the wild-type, ced-6(n2095), ced-5(n1812), and ced-10(n1993) 559 mutant embryos are from Figs $1 \mathrm{E}$ and $9(\mathrm{~A}-\mathrm{C})$, respectively. (I) The data for the wild-type, ced-

560 6(n2095), ced-5(n1812), and ced-10(n1993) mutant embryos are from Figs $1 \mathrm{H}$ and 9(F-H), 561 respectively.

562 (E and $J)$ Box-and-Whiskers plots of the relative mCherry signal intensity measured in the center of 563 phagosomes 50 min-post the formation of nascent C3 phagosomes from 15 each of wild-type, ced564 6(n2095), ced-5(n1812), and ced-10(n1993) mutant embryos. The Red dashed lines indicate where 565 value 1, which represents no signal enrichment relative to the background signal, stand. “***”, $p<0.001$; 566 ns, not significant, Student $t$-test against the wild-type samples.

567 (K) A diagram illustrating that between the two parallel pathways that regulate the clearance of apoptotic cells, only the CED-1 pathway, but not the other pathway, plays an essential role in promoting the incorporation of autophagosomes into phagosomes. Blue letters in parentheses are the names of the mammalian homolog of the corresponding C. elegans proteins.

\section{The incorporation of lysosomes into phagosomes is not affected by the lack of autophagosomes}

The incorporation of lysosomes to phagosomes is an essential force that drives the degradation of phagosomal contents [3]. Because a portion of the autophagosomes would fuse with lysosomes and become autolysosomes that retain lysosomal features [9], it is possible that the $\mathrm{LGG}^{+}$particles, which include both autophagosomes and autolysosomes, might act to provide additional vesicles bearing lysosomal features (such as autolysosomes) to the phagosomes. To test whether this is the case, we examined whether mutations that specifically impair autophagosomes biogenesis would affect the amount of organelles with lysosomal features, including lysosomes and autolysosomes that are incorporated into phagosomes, and the temporal dynamics of the incorporation. C. elegans NUC-1 is an endonuclease belonging to the DNase II family and resides in the lysosomal lumen [43,44]. We used a nuc-1::mCherry reporter expressed in engulfing cells to quantify the rate of lysosome-phagosome fusion. In time-lapse recording images monitoring phagosomes containing cell corpses $\mathrm{C} 1, \mathrm{C} 2$, and $\mathrm{C} 3$ in wild-type embryos, we observed the attachment of NUC-1::mCherry puncta on the surfaces of phagosomes and the subsequent accumulation of the mCherry signal into the phagosomal lumen (Fig 10A). This dynamic process represents the recruitment and the subsequent fusion of lysosomal particles to phagosomes and the delivery of NUC-1::mCherry into the phagosomal lumen. We next measured the 
588 signal level of NUC-1::mCherry in the center of phagosomes in $\operatorname{lgg}-1(\mathrm{tm} 3489), \operatorname{lgg}-2(\mathrm{tm} 5755)$, and atg7(bp411) embryos over time and observed accumulation of mCherry signal in the phagosomal lumen in

590 all samples (Fig 10 A-E). These three mutants are defective in the biogenesis of autophagosomes.

591 Quantitative analysis of 15 phagosomes of each genotype found that, $60 \mathrm{~min}$ after the formation of the 592 nascent phagosomes, (1) no significant difference in the mCherry signal level in the phagosomal lumen 593 in lgg-2 mutants compared to wild-type samples (Fig 10F), and (2) slight elevation of the mCherry 594 signal in the lumen of $l g g-1$ and atg-7 mutant samples. In addition, the median value of the first time point when NUC-1::mCherry signal was detected inside the phagosomal lumen was not significantly

596 different in all four genotypes (Fig 10G). All these data demonstrate that the incorporation of 597 lysosomes into phagosomes in $\operatorname{lgg}-1, \operatorname{lgg}-2$, and $\operatorname{atg}-7$ mutants is at least as efficient as in wild-type embryos. Therefore, defects in the formation of autophagosomes, which potentially would result in the lack of autolysosomes, do not affect the incorporation of lysosomes into phagosomes.

600

A

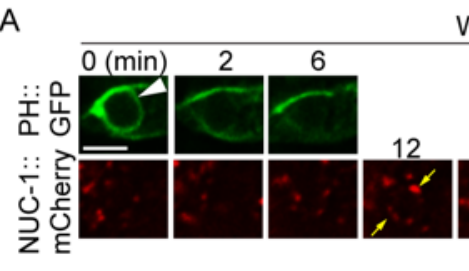

B

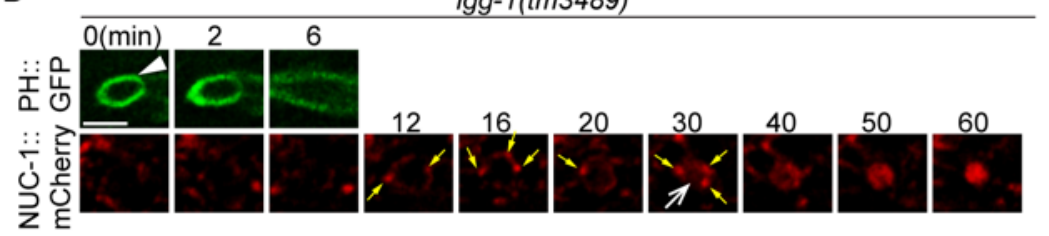

C

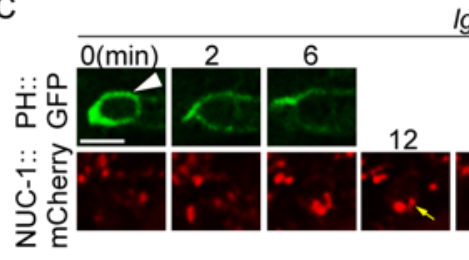

D

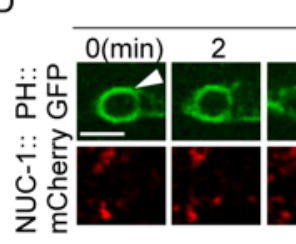

Wild-type

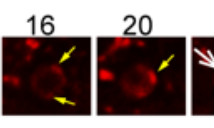

$\operatorname{lgg}-2(\operatorname{tm} 5755)$

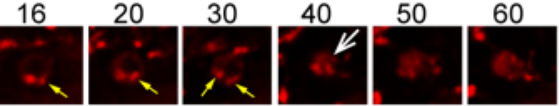

atg-7(bp411)

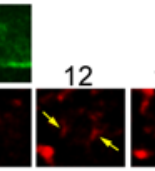

$\mathrm{E}$

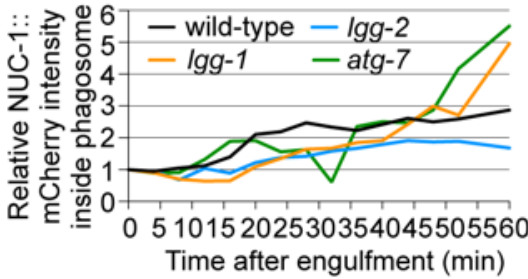

$\mathrm{F}$

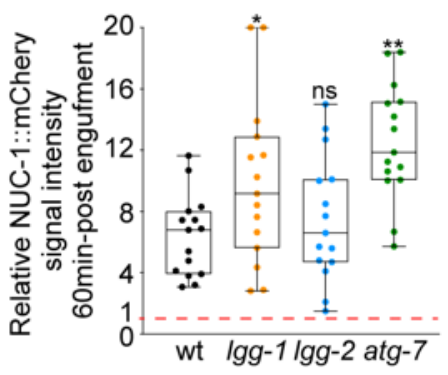

\section{(}

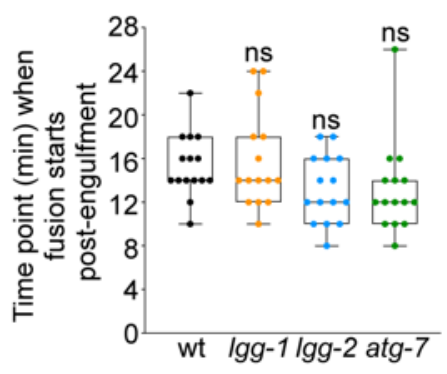

601

602

603

604

605

Figure 10. Defects in the formation of autophagosomes do not affect the timing or efficiency of lysosomes incorporation into phagosomes

The time-lapse recording was conducted on phagosomes containing $\mathrm{C} 1, \mathrm{C} 2$, and $\mathrm{C} 3$ in wild-type and named mutant embryos carrying $\mathrm{P}_{c e d-1} n u c-1:$ :mCherry, the lysosomal lumen reporter, and $\mathrm{P}_{\text {ced- }}$ 
$606{ }_{1} P H(P L C Y):: g f p$, the marker for extending pseudopods and nascent phagosomes. (A-D) Fluorescence

607 time-lapse images of one C3 phagosome in each strain with the indicated genotype. "0 min" is the

608 moment when a nascent phagosome (white arrowhead) just seals. Yellow arrows mark the lysosomal

609 particles that are located on phagosomal surfaces. White open arrows mark the phagosomes with

610 mCherry signals in the lumen. Scale bars are $2.5 \mu \mathrm{m}$.

611 (E) The relative NUC-1::mCherry signal intensity in the center of a phagosome (Y-axis) over time (in 2612 min interval) (X-axis). "0 min" indicates the moment when a nascent phagosome is just sealed. Data 613 are from Fig $10(A-D)$.

614 (F) A Box-and-Whiskers graph of the relative mCherry signal intensity measured in the center of 615 phagosomal lumen 60 min-post the formation of nascent C3 phagosomes from 15 each of wild-type, 616 Igg-1(tm3489), Igg-2(tm5755), and atg-7(bp411) mutant embryos. A dashed red line marks the position 617 where the relative signal intensity value $=1$, which indicates no enrichment of mCherry signal inside the 618 phagosomal lumen.

619 (G) A Box-and-Whiskers graph displaying the first time point when the NUC-1::mCherry signal is 620 detected inside the lumen of 15 C3 phagosomes in wild-type, lgg-1(tm3489), lgg-2(tm5755), and atg7(bp411) mutant embryos.

\section{Discussion}

Autophagosomes play essential roles in cellular homeostasis by eliminating harmful protein aggregates and damaged organelles, and in stress response by converting intracellular organelles to nutrients during starvation [9]. We have identified a novel function of autophagosomes in facilitating the degradation of apoptotic cells through fusing to phagosomes. In C. elegans cells that engulf apoptotic cells, autophagosomes are recruited to the surfaces of phagosomes and subsequently fuse to the phagosomal membrane. Through this previously unknown interaction, autophagosomes release substances to the phagosomal lumen and membrane. We have further identified a signaling pathway that promotes the recruitment and subsequent fusion of autophagosomes to phagosomes. Together, these findings reveal a novel mechanism through which the pathways that control autophagy and phagocytosis converge, underlining the importance of this mechanism in the degradation of apoptotic cells. We propose that this mechanism might be conserved in the metazoans and has important impact 
Previously, $C$. elegans autophagy genes have been implicated in facilitating the clearance of apoptotic cells. Autophagy occurring in apoptotic cells was proposed to facilitate the exposure of phosphatidylserine, which is the "eat me" signal that attracts engulfing cells to the surfaces of apoptotic cells in mice embryonic bodies and C. elegans $[45,46]$. Multiple autophagy genes were reported to participate in the clearance of apoptotic cells in $C$. elegans engulfing cells $[11-13,46]$. They were reported to work together with Class II PtdIns3 kinase PIKI-1 to facilitate the production of PtdIns(3)P on phagosomal surfaces [11], to promote the recruitment of RAB-5 and RAB-7 to the phagosomal surfaces [13], and to promote phagosome degradation [46]. The above reports suggest that the autophagy genes examined might have separate functions in the degradation of phagosomal contents not related to their canonical roles in autophagosome biogenesis. Our work reported here, on the other hand, presents a different discovery regarding how the autophagy machinery regulates phagosome degradation.

We found that the canonical double-membrane autophagosomes actively participate in the degradation of apoptotic cells inside phagosomes. Four critical sets of evidence indicate that the LGG1- and -2-labeled vesicles that are incorporated into phagosomes are autophagosomes, not the singlemembrane LAP vesicles. First, the membrane-bound LGG-1 or -2 reporters were observed to enter the phagosomal lumen after vesicle-phagosome fusion. Only when the vesicles are composed of double membranes and when the reporter molecules label both the inside and outside membranes, the entry of the signal into phagosomal lumen is possible (Fig 1K). Conversely, if the LGG-1 or -2 labeled vesicles are of a single membrane, after vesicle-phagosome fusion, the reporter signal would remain on the phagosomal membrane, as the membrane-bound lysosomal marker CTNS-1::mRFP does (Fig S1). Secondly, these vesicles are also labeled with GFP-tagged ATG-9, an integral membrane protein inserted into the autophagosomal membranes. Thirdly, the production of these vesicles relies on genes pivotal for autophagosome biogenesis, such as atg-3, atg-7, and atg-9. Last but not least, genes encoding the $C$. elegans homologs of mammalian ULK1, ATG13, and ATG14, which are dispensable for the generation of LAP vesicles in mammalian cells [6], are required for the formation of the LGGlabeled vesicles discussed here and for the efficient clearance of apoptotic cells, further verifying that these vesicles are distinct from the single-membrane LAP vesicles. Together, these four sets of findings demonstrate that double-membrane autophagosomes, not single-membrane LAP vesicles, are being incorporated into phagosomes.

In C. elegans, LC3-dependent phagocytosis was reported to function in the clearance of the midbody, a structure that is essential for the completion of cytokinesis, and the second polar body 
670 generated during female meiosis $[47,48]$. The clearance of midbodies and second polar body is

671 considered independent of autophagosomes based on the observation that neither unc-51 nor epg-8 is

672 required for the above processes [47,48]. However, since whether the LGG markers attached to

673 phagosomes carrying midbodies or polar bodies represent double-membrane or single-membrane

674 vesicles have not been investigated [47,48], whether LAP vesicles exist in C. elegans cells still needs to

675 be demonstrated.

676 The observation of the LGG-1 or -2 signals inside the phagosomal lumen is shown through the

677 presence of mCherry as a reporter molecule. The mCherry protein is resistant to acidic $\mathrm{pH}$ [30].

678 Phagosome luminal $\mathrm{pH}$ value reduces from 5.5-6.0 to 4.5-5.5 after incorporating lysosomes [49], leading

679 to the inactivation of the fluorophore in the commonly used GFP reporter [29]. In fact, in C. elegans

680 engulfing cells, GFP-tagged LGG-1 or -2, unlike the mCherry-tagged reporters, are only observed on the

681 surfaces but not in the lumen of phagosomes, presumably due to the acidic environment of the

682 phagosomal lumen (Fig 1). It is thus conceivable that in other experimental systems, a mCherry-tagged

683 LC3 marker might disclose a previously overlooked fusion between autophagosomes and phagosomes.

Based on the evolutionary conservation of almost all molecular mechanisms, in particular the strong conservation of the known mechanisms that control phagocytosis and autophagy in metazoans $[25,34]$, we propose that in in mammalian cells, besides LAP vesicles, canonical autophagosomes might also contribute to the degradation of apoptotic cells in phagosomes. The demonstration of an

autophagosome-phagosome fusion event in C. elegans, a well-established model organism for the study of cell death-related events, opens a path to investigate whether the event and mechanism of autophagosome-phagosome fusion is an evolutionarily conserved event. In addition, the autophagosome-phagosome fusion might contribute to the degradation of other kinds of phagosomal cargos besides apoptotic cells.

\section{LGG-1 and LGG-2 define three subpopulations of autophagosomes that are incorporated into} phagosomes

LGG-1 and -2 are close homologs that each labels autophagosomes. We have observed three separate classes of autophagosomes that are incorporated into phagosomes: LGG-1 ${ }^{+}$LGG-2, ${ }^{-}$GG-1 ${ }^{-}$ LGG-2 ${ }^{+}$, or LGG- ${ }^{+}$LGG-2 ${ }^{+}$. These subpopulations might represent autophagosomes at different stages of maturation [26]. Remarkably, we have found that the $\operatorname{lgg}-1$; $\operatorname{lgg}-2$ double mutants display a much enhanced Ced phenotype than that displayed by each of the lgg-1 or lgg-2 single mutants. Together, these results indicate that autophagosomes at different stages of maturity all contribute to the 
degradation of apoptotic cells. These results are also consistent with our finding that the autophagosomes-phagosome fusion is independent of lysosome-phagosome fusion (Fig 9).

\section{What do autophagosomes contribute to the degradation of apoptotic cells}

We have found that the blockage of the biogenesis of autophagosomes or the recruitment of autophagosomes to phagosomes result in a significant delay in the degradation of phagosomal content and, consequently, the persistent appearance of engulfed cell corpses. The incorporation of autophagosomes into phagosomes presumably delivers certain substances to the lumen and/or membrane of phagosomes, substances that are important for the degradation of phagosomal contents. Currently, the identities of these substances are still a mystery. Studies in mammalian cells implicate LAP vesicles in facilitating the incorporation of lysosomes into phagosomes [5,6]. In C. elegans mutants defective for the biogenesis of autophagosomes (atg-7, lgg-1, and lgg-2 mutants), we found that the lack of autophagosomes does not affect the efficiency of lysosome-phagosome fusion (Fig 10). This result, together with the observation that the three subpopulations of autophagosomes at different maturation stages all fuse to phagosomes, suggests that it is not just the sub-population of autolysosomes, which have lysosomal features, that contribute to phagosomal degradation. Thus autophagosomes might deliver something unique to the phagosome, something not present in lysosomes or endosomes. These substances also might not be limited to proteins in the autophagosome lumen or membranes; they could be specific lipid molecules from the membranes of autophagosomes. Further investigation is required to discover the identities of this (these) molecule(s).

\section{The CED-1 signaling pathway drives the incorporation of autophagosomes to phagosomes}

Autophagosomes are incorporated into phagosomes in two sequential steps: (1) they are recruited to the surfaces of phagosomes, detected by the enrichment of punctated LGG-1 or -2 fluorescence reporters on phagosomal surfaces, and (2), they subsequently fuse to phagosomes, detected by the enrichment of the LGG-1 or -2 reporter signals inside the phagosomal lumen. RAB-7 and its effector, the HOPS complex, are known to act as tethering factors that facilitate the fusion of various intracellular organelles, including autophagosomes, to lysosomes [26,50]. Closely related to this study, RAB-7 and VPS-18 are pivotal for the fusion between lysosomes and phagosomes and for the degradation of apoptotic cells inside phagosomes in C. elegans [19,42,51]. Here we have discovered that RAB-7 and VPS-18 play essential and specific roles in the fusion but not for the recruitment of autophagosomes to 
734 phagosomes. Our finding adds a new pair of organelles that depend on the RAB-7/HOPS complex for 735 fusion to each other. As RAB-7 is enriched on the surfaces of both phagosomes and autophagosomes 736 ([19] and (Fig 7(B-D)), and as the HOPS complexes in mammalian cells and Drosophila are known to 737 interact with the SNARE complex, the membrane fusion machinery [52,53], we propose that the RAB-

738 7/HOPS complex acts on the surfaces of phagosomes and autophagosomes to facilitate autophagosomes739 phagosome fusion via promoting the interaction between the SNARE complexes on phagosomes and 740 autophagosomes (Fig 7I). Currently, the specific SNARE proteins that catalyze the autophagosomes741 phagosome fusion have not been identified.

742 The CED-1 signaling pathway, which initiates the maturation of phagosomes that bear apoptotic 743 cells, is essential for the enrichment of GTP-bound RAB-7 to the surfaces of phagosomes [19]. Here we 744 have found that CED-1, CED-6, and DYN-1 drive the incorporation of autophagosomes to phagosomes, 745 in addition to driving the incorporation of early endosomes and lysosomes previously discovered 746 [19,21]. In ced-1 mutants, for example, the recruitment of autophagosomes to the surfaces of 747 phagosomes is almost completely blocked. Due to the severe recruitment defect, whether the ced-1 null mutation further impairs the fusion between autophagosomes and phagosomes cannot be readily visualized. However, since a ced-1 null mutation impairs the recruitment of RAB-7 to [19] and the production of PtdIns(3)P on phagosomal surfaces [19,23], and since the recruitment of the HOPS complex to the surfaces of intracellular organelles requires both RAB7 and PtdIns(3)P [39,54,55], we predict that the CED-1 pathway would also control the RAB-7/HOPS complex-mediated autophagosomes-phagosome fusion (Fig 9K). Identifying the CED-1 signaling pathway as the driving force for the incorporation of autophagosomes to phagosomes helps to reveal the molecular mechanisms behind the crosstalk between autophagy and phagocytosis.

\section{Materials and Methods}

\section{Mutations, strains, and transgenic arrays}

C. elegans strains were grown at $20^{\circ} \mathrm{C}$ as previously described [56] unless indicated otherwise.

760 The N2 Bristol strain was used as the wild-type control strain. Mutations are described in [57] and by 761 the Wormbase (http://www.wormbase.org) unless noted otherwise (Table S1): LG1: ced-1(e1735), epg762 8(bp251); LGII: lgg-1(tm3489), rab-7(ok511), vps-18(tm1126); LGIII: atg-13(bp414), ced-6(n2095); 763 LGIV: atg-3(bp412), atg-7(bp411), ced-5(n1812), ced-10(n1993), lgg-2(tm5755 and tm6474); LGV: 764 atg-9(bp564), atg-18(gk378), unc-76(e911), unc-51(e369); LGX: atg-2(bp576), dyn-1(n4039). dyn7651 (n4039) homozygous mutants, which are zygotic embryonic lethal, were maintained by an 
extrachromosomal array carrying a wild-type $d y n-1$ gene and a co-expressed $\mathrm{P}_{\text {egl-13gfp }}$ marker (Table S1) [21]. dyn-1(n4039) homozygous embryos losing the rescuing transgene were identified as the

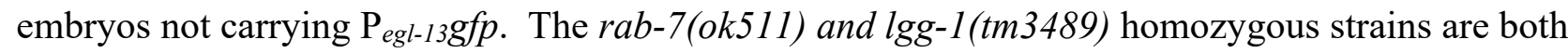
maternal-effect embryonic lethal, and the mIn1 balancer maintained each allele with an integrated pharyngeal GFP marker (Table S1) [58]. To obtain rab-7(ok511) mz' homozygous embryos, GFP- rab7(ok511) homozygous hermaphrodites were isolated among the progeny of the strain VC308, and their progeny were collected as embryos. The same protocol was used to collect $\operatorname{lgg}-1(\mathrm{tm} 3489) \mathrm{m}^{-} \mathrm{z}^{-}$embryos from the strain GK738 (Table S1). Double mutants between $\operatorname{lgg}-1(\operatorname{tm} 3489) / \mathrm{mIn} 1$ and $\operatorname{lgg}$-2(tm6474) were generated by standard genetic crosses.

Extrachromosomal arrays were generated by the microinjection of plasmids with the co-injection marker p76-18B [punc-76(+)] into unc-76(e911) mutants $[59,60]$. Non-Unc mutants were identified as transgenic animals. Integrated transgenic arrays were generated by gamma irradiation [60]. Integrated arrays generated in this study are as follows (Table S1): LGI: enIs87[P $P_{c e d-1} P H(h P L C \gamma):: m r f p$ and $P_{c e d-}$ ImNG::lgg-1]; LGII: enIs82[P $P_{\text {ced-1 }}$ ced-1::gfp and $P_{\text {ced-1 }}$ mCherry::lgg-1]; LGV: enIs85[P ced-1 $_{\text {ing }}$ PH(hPLC $\gamma):: m r f p$ and $\left.P_{c e d-1} m N G:: \operatorname{lgg}-2\right]$; LGX: enIs83[P $P_{\text {ced-1 }}$ ced-1::gfp and $P_{\text {ced-1 }} m$ Cherry::lgg-2].

\section{Plasmid construction}

lgg-1 and lgg-2 cDNAs were PCR amplified from a mixed-stage C. elegans cDNA library [61]. To generate the $\mathrm{P}_{c e d-1} g f p:: \operatorname{lgg}-1$ and $\mathrm{P}_{c e d-1} g f p:: \operatorname{lgg}-2$ plasmids, $\operatorname{lgg}-1$ and $\operatorname{lgg}-2$ cDNAs were cloned into the XmaI and KpnI sites of plasmid pZZ956 ( $P_{\text {ced-1 }} 5$ 'gfp) [61]. $\mathrm{P}_{\text {ced-1 }}$ mCherry::lgg-1 or -2 were constructed by replacing the cDNA of $g f p$ with that of mCherry [30]. $\mathrm{P}_{\text {ced-1 }} m N G$ (mNeonGreen)::lgg-1 or -2 were constructed by replacing the cDNA of $g f p$ with that of mNeonGreen [62]. To generate $\mathrm{P}_{c e d-}$ Igfp::lgg-1(G116A) and $\mathrm{P}_{c e d-1} g f p:: \operatorname{lgg}-2(G 130 A)$, the QuickChange Site-directed Mutagenesis Kit (Stratagene, La Jolla, CA) was used to introducing the above mutations into the constructs. To construct $\mathrm{P}_{\text {ced-1 }}$ atg-9:: $m$ Cherry, the atg-9a open reading frame was PCR-amplified from a $C$. elegans mixed-stage cDNA library and inserted between $\mathrm{P}_{c e d-1}$ and $g f p$ in pZZ829 ( $\mathrm{P}_{c e d-1} 3$ 'gfp) [61]. The $g f p$ cDNA was then replaced by the $m$ Cherry cDNA [30]. All plasmids contain an unc-54 3' UTR.

\section{Quantification of the number of cell corpses using Nomarski DIC microscopy}

Cell corpses display a highly refractive button-like morphology under Differential Interference Contrast (DIC) microscopy. An Axionplan 2 compound microscope (Carl Zeiss, Thornwood, NY) equipped with Nomarski DIC optics, an AxioCam digital camera, and AxioVision imaging software was 
used for DIC microscopy. Using a previously established protocol [27], we quantified the number of cell corpses in the head region at the 1.5-fold and 2-fold stage embryos, which are $\sim 420$ and $\sim 460 \mathrm{~min}$ post-first cleavage.

\section{Fluorescence microscopy and time-lapse imaging}

A DeltaVision Elite Deconvolution Imaging System (GE Healthcare, Inc.) equipped with a DIC imaging apparatus and a Photometrics Coolsnap 2 digital camera was used to capture fluorescence, and DIC images Applied Precision SoftWoRx 5.5 software was utilized for deconvolving and analyzing the images [27]. To observe the amount of autophagosomes in ventral hypodermal cells that express $\mathrm{P}_{c e d-1}$, fourteen serial Z-sections in $0.5 \mu \mathrm{m}$ interval between adjacent optical sections, starting at the ventral surface of embryos at mid-embryonic stages, were collected. The 2D projection image of each Z-stack was generated and compared among different genetic backgrounds (Figs S1, S2, S4, and S6). To track fluorescence markers on pseudopods, on the surfaces of phagosomes, or inside phagosomal lumen during the clearance process of cell corpses $\mathrm{C} 1, \mathrm{C} 2$, and $\mathrm{C} 3$, embryos were monitored on their ventral surface starting at $\sim 310$ min post-first cleavage using an established time-lapse imaging protocol [27]. Twelve to 16 serial $Z$-sections (at 0.5 - $\mu \mathrm{m}$ intervals) were captured every $2 \mathrm{~min}$, with recordings typically lasting between 60 to 180 min. Embryos that exhibited normal elongation and movement were considered developing properly. The moment engulfment starts is defined as when the extension of pseudopods around $\mathrm{C} 1, \mathrm{C} 2$, or C3 is first observed. The moment a nascent phagosome form is defined as when the pseudopods around a cell corpse join and make a full closure. The life span of a phagosome is defined as the time interval between the moments when the nascent phagosome forms and when the phagosome shrinks to one-half of its original diameter.

The time spans of the engulfment and degradation processes of cell corpses $\mathrm{C} 1, \mathrm{C} 2$, or $\mathrm{C} 3$ were measured as previously established [61]. Briefly, a pseudopod marker, either CED-1::GFP or PH(PLC $\gamma):: G F P$, was monitored over time. The moment engulfment starts is defined as when the budding pseudopods around $\mathrm{C} 1, \mathrm{C} 2$, or $\mathrm{C} 3$ is first observed. The moment a nascent phagosome form is defined as the moment when the pseudopods around a cell corpse join and make a full closure. The period between the budding and the sealing of the pseudopods is the time span of engulfment. To measure phagosome duration, a co-expressed phagosomal surface marker mCherry::2xFYVE was used to track the diameter of the phagosome over time. The life span of a phagosome is defined as the time interval between the time points when a nascent phagosome was born and when the phagosome shrank to one-half of its original diameter. 


\section{Quantitative measurement of signal intensity}

Measuring the signal intensity inside the phagosomal lumen

In embryos expressing mCherry- or mNG-tagged LGG-1 or LGG-2, to measure the fluorescence signal intensity inside the phagosomal lumen over time, we identified the boundary of a phagosome and the " 0 min" time point when a nascent phagosome was formed by observing the co-expressed marker for a nascent phagosome such as CED-1::GFP or PH(PLC $\gamma):: G F P$. At each time point, the total LGG-1 or 2 image intensity of a fixed area (4x4 pixels) at the center of a phagosome (Int phagosome) was recorded (Tn) comparing to the start point (T0) is calculated as RInt $\mathrm{Tn}=\left(\mathrm{Int}_{\text {phagosome- }} \mathrm{Int}_{\text {background }}\right)_{\mathrm{Tn}} /\left(\mathrm{Int}_{\text {phagosome }}\right.$ into the phagosomal lumen.

\section{Measuring the signal intensity on the surface of a phagosome}

To measure the efficiency of recruitment of autophagosomes to phagosomes, we quantified the intensity of mCherry- or mNG-labeled LGG-1 or LGG-2 on the surfaces of phagosomes. First, we identified the boundary of a phagosome and the " 0 min" time point when a nascent phagosome just intensities, as well as the areas of the polygons, were recorded. The unit signal intensity of the "donutshape" area between the two polygons was calculated as follows: polygon). The Unit Background Intensity (UI background) was measured from a polygon outside the embryo was calculated as follow: $\mathrm{UI}_{\text {background }}=$ Intensity background $/$ Areabackground.

At the time point Tn, the relative signal intensity $\left(\mathrm{RInt}_{\mathrm{Tn}}\right)=\left(\mathrm{UI}_{\mathrm{phagosome}}-\mathrm{UI}_{\text {background }}\right)_{\mathrm{Tn}} /\left(\mathrm{UI}_{\mathrm{phagosome}}-\right.$

$\left.\mathrm{UI}_{\text {background }}\right)_{\mathrm{T} 0}$. The RInt $\mathrm{Tn}_{\mathrm{T}}$ value 1.0 indicates no enrichment of LGG-1- or LGG-2-labeled

\section{Acknowledgment}

We thank Ying Wang and Lidan Gao for technical support and R. Haley for helpful comments. autophagosomes on phagosomal surfaces comparing to the " 0 min" time point. 
861 Programs (P40 OD010440), for providing some strains. We also thank the National BioResource

862 Project of Japan and Dr. Shohei Mitani for provides some strains.

863

864 Funding Source

865 NIH R01GM067848 supports this work.

866

867 Author Contributions

868 The authors have made the following declarations about their contributions: Conceived and

869 designed the experiments: OPR ZZ. Performed the experiments: OPR LC XL TY HH ZZ. Analyzed

870 the data: OPR TY ZZ. Prepare the manuscript: OPR ZZ.

871

872 Competing Interests

873

The authors declare no competing interests 
A

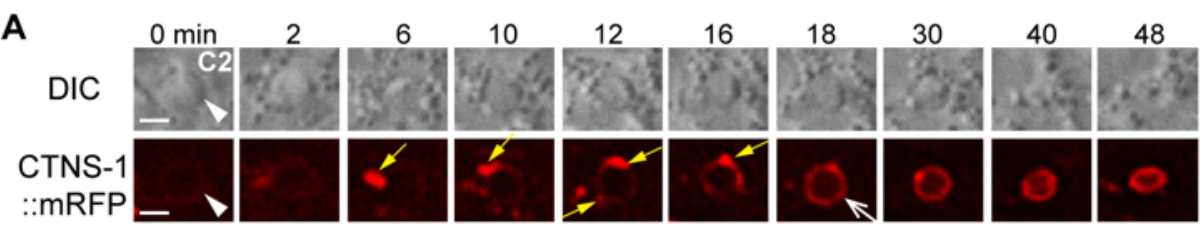

B

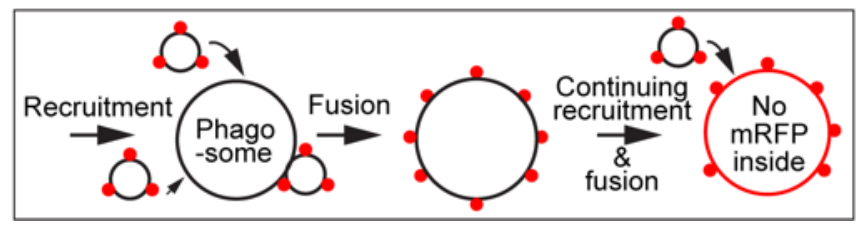

Figure S1. The fusion of lysosomal particles to phagosomes results in the incorporation of Iysosomal membrane protein CTNS-1 to the phagosomal membrane but not the lumen
(A) Time-lapse images of a C2 phagosome and the lysosomes that are recruited to and subsequently fuse with it. CTNS-1::mRFP is expressed in engulfing cells under $\mathrm{P}_{\text {ced-1. }}$. 0 in" is the time point when a C2 phagosome (white arrowheads) just forms. Yellow arrows mark CTNS-1::mRFP-labeled lysosomal particles. One open white arrow marks the phagosomal surface that is evenly colored by mRFP. Scale bars are $2 \mu \mathrm{m}$.
(B) A diagram illustrating that the single membrane-vesicles that are labeled with CTNS-1::mRFP on their membranes are recruited to phagosomal surfaces and fused to the phagosomal membrane. After the fusion between the membranes of these vesicles and the phagosome, the mRFP signal is evenly distributed to the phagosomal surface. However, no mRFP signal enters the phagosomal lumen. 
A

B

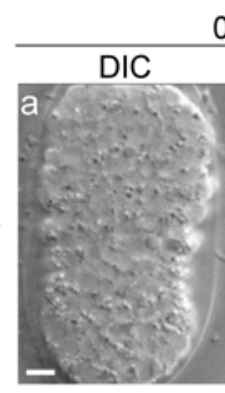

0 (min)
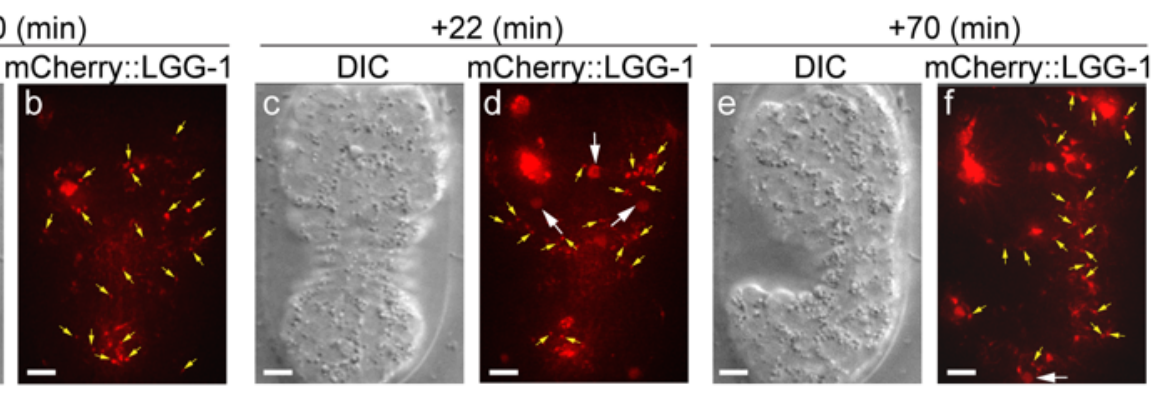

Wild-Type
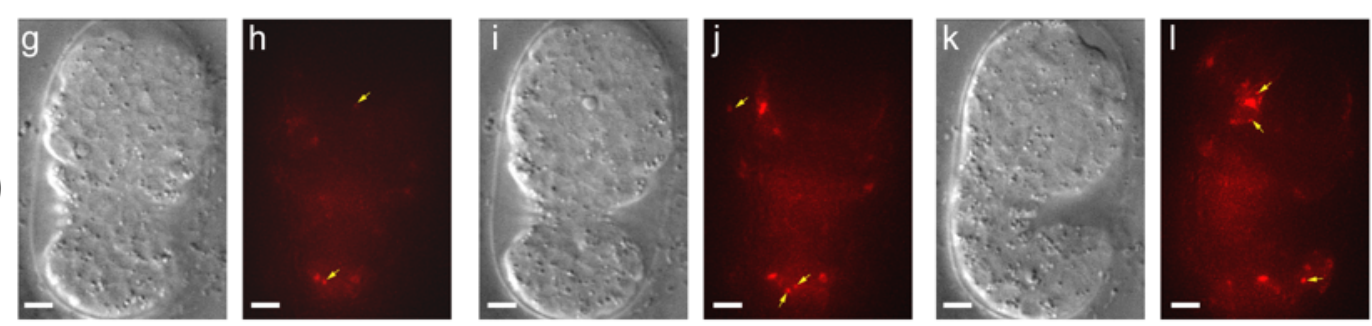

C
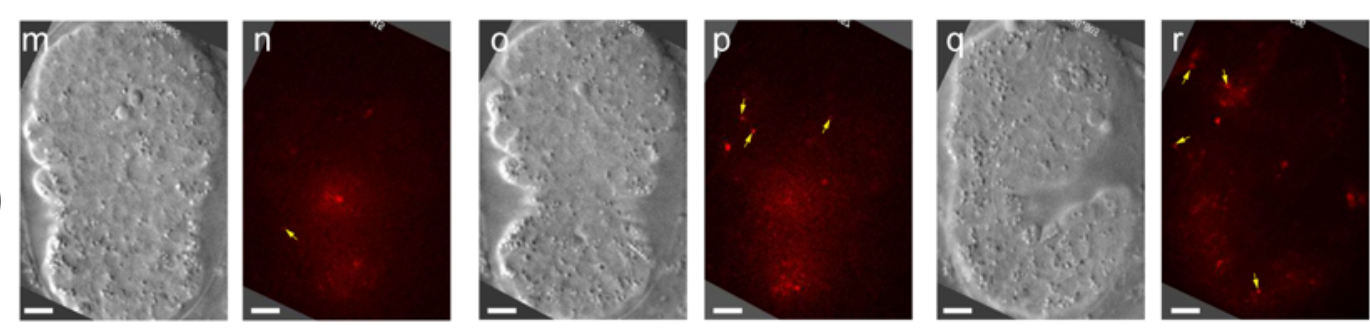

D
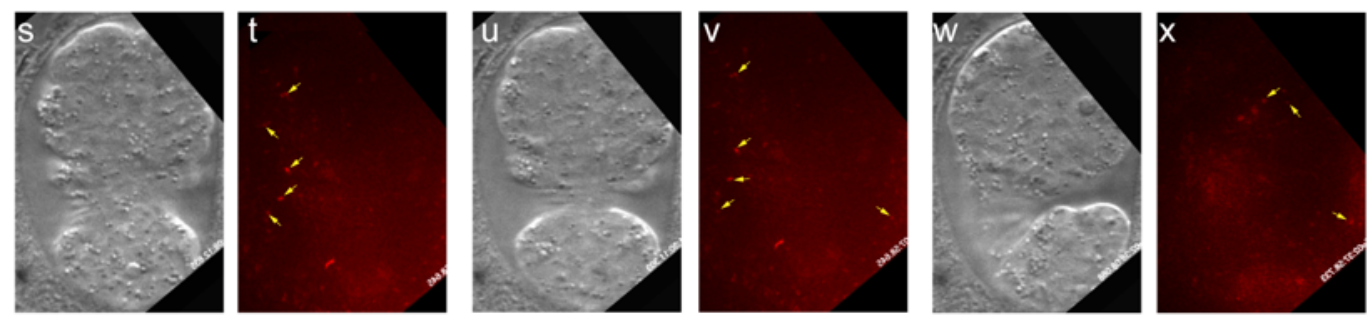

Figure S2. The atg-7, atg-13, and epg-8 mutants are severely defective in the production of LGG-

\section{1-labeled autophagosomes}

895 Images of wild-type (A), atg-7(bp411) (B), atg-13(bp414) (C), and epg-8(bp251) mutant (D) embryos

896 expressing $\mathrm{P}_{c e d-1} m$ Cherry::Igg-1 are presented. "0 min" labels embryos that are $\sim 330$ min post- $1^{\text {st }}$

897 cleavage. The mCherry images are 2-D projections of 14 Z-sections at $0.5 \mu \mathrm{m}$ intervals. Red puncta

898 (yellow arrows) represent autophagosomes. White arrows in $(A(d))$ mark three phagosomes, on the

899 surface of which mCherry signal is recruited in (a-b), and 20 min later, enters the phagosome,

900 demonstrating the autophagosomes/phagosome fusion event. Scale bars are $5 \mu \mathrm{m}$. 
A

Wild-Type

atg-7

(bp411)

B

c

atg-13

(bp414)

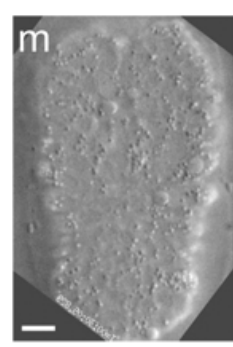

D

epg-8

(bp251)
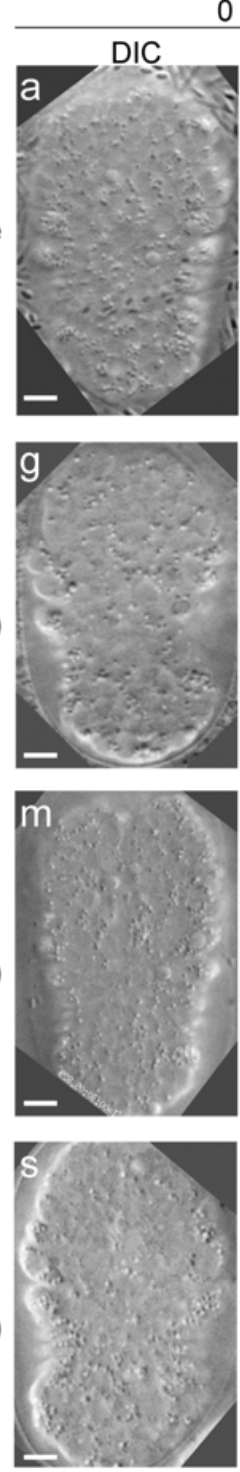

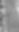

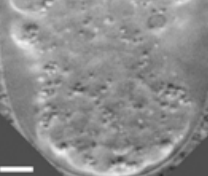

0 (min)
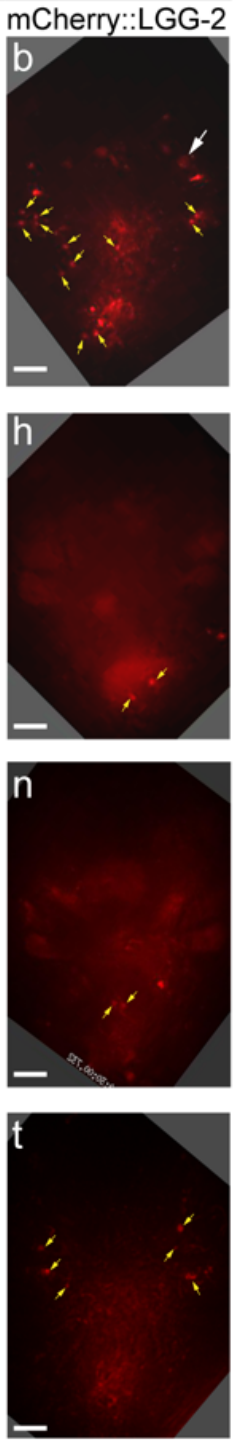
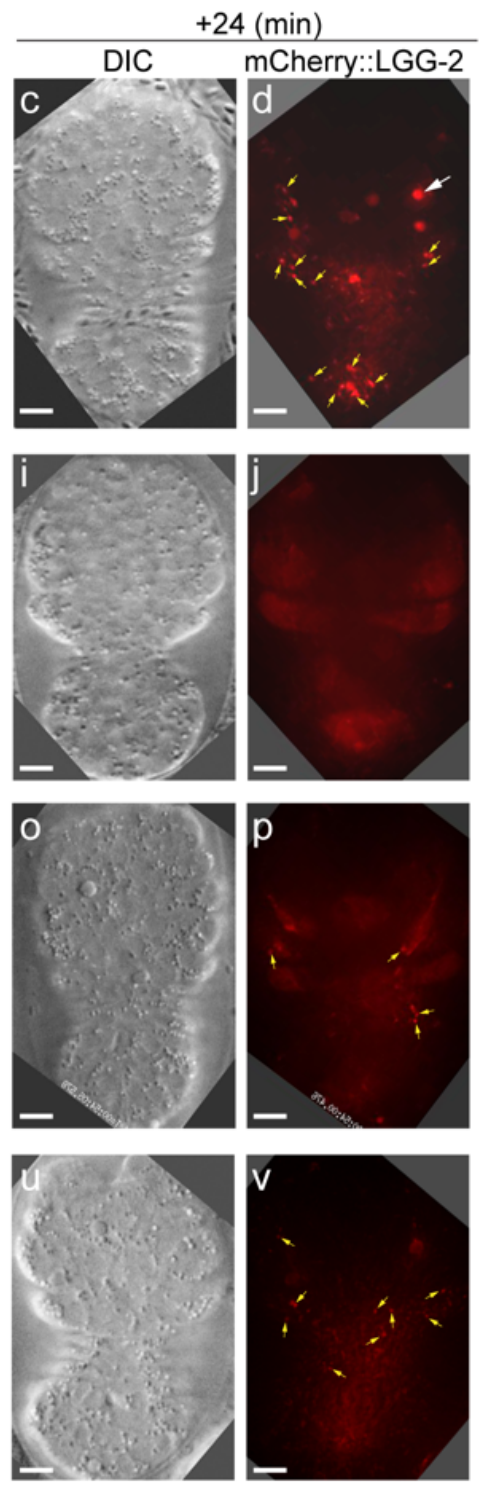
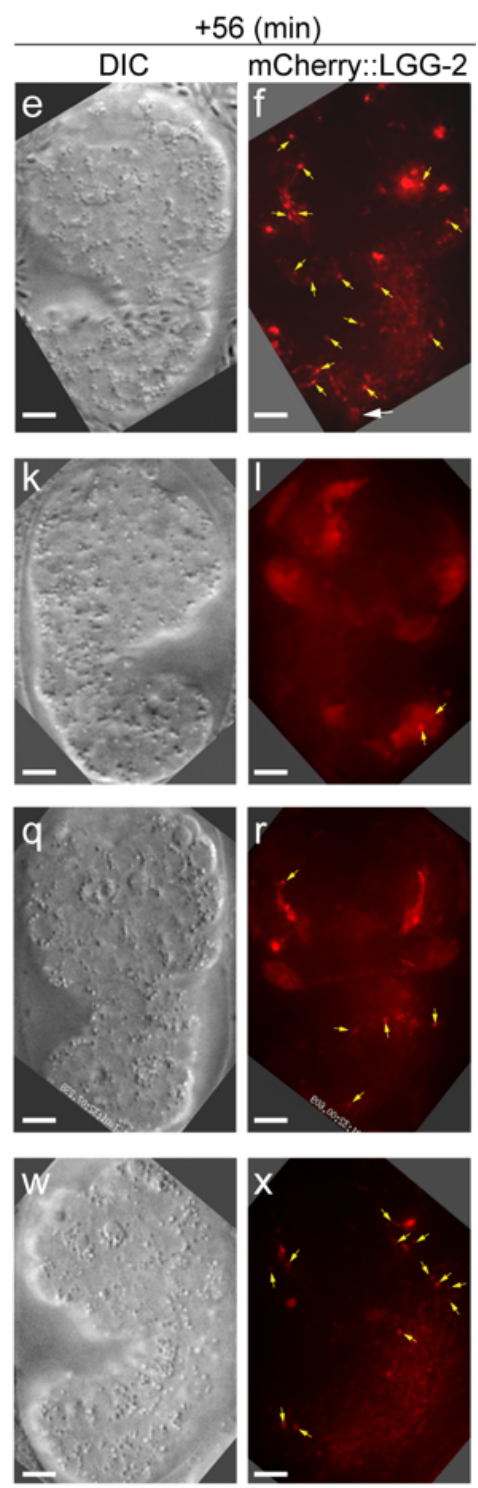

904 Figure S3. The atg-7, atg-13, and epg-8 mutants are severely defective in the production of LGG905 2-labeled autophagosomes

906 Images of wild-type (A), atg-7(bp411) (B), atg-13(bp414) (C), and epg-8(bp251) mutant (D) embryos

907 expressing $\mathrm{P}_{\text {ced-1 }}$ mCherry::Igg-1 are presented. "0 min" labels embryos that are $\sim 330$ min post- $1^{\text {st }}$

908 cleavage. The mCherry images are 2-D projections of 14 Z-sections at $0.5 \mu \mathrm{m}$ intervals. Red puncta

909 (yellow arrows) represent autophagosomes. White arrows in $(A(b, d))$ mark one phagosome, on the

910 surface of which mCherry puncta are recruited to in (b), and 24 min later, mCherry enters the

911 phagosome, demonstrating the autophagosomes/phagosome fusion event. Scale bars are $5 \mu \mathrm{m}$.

912 

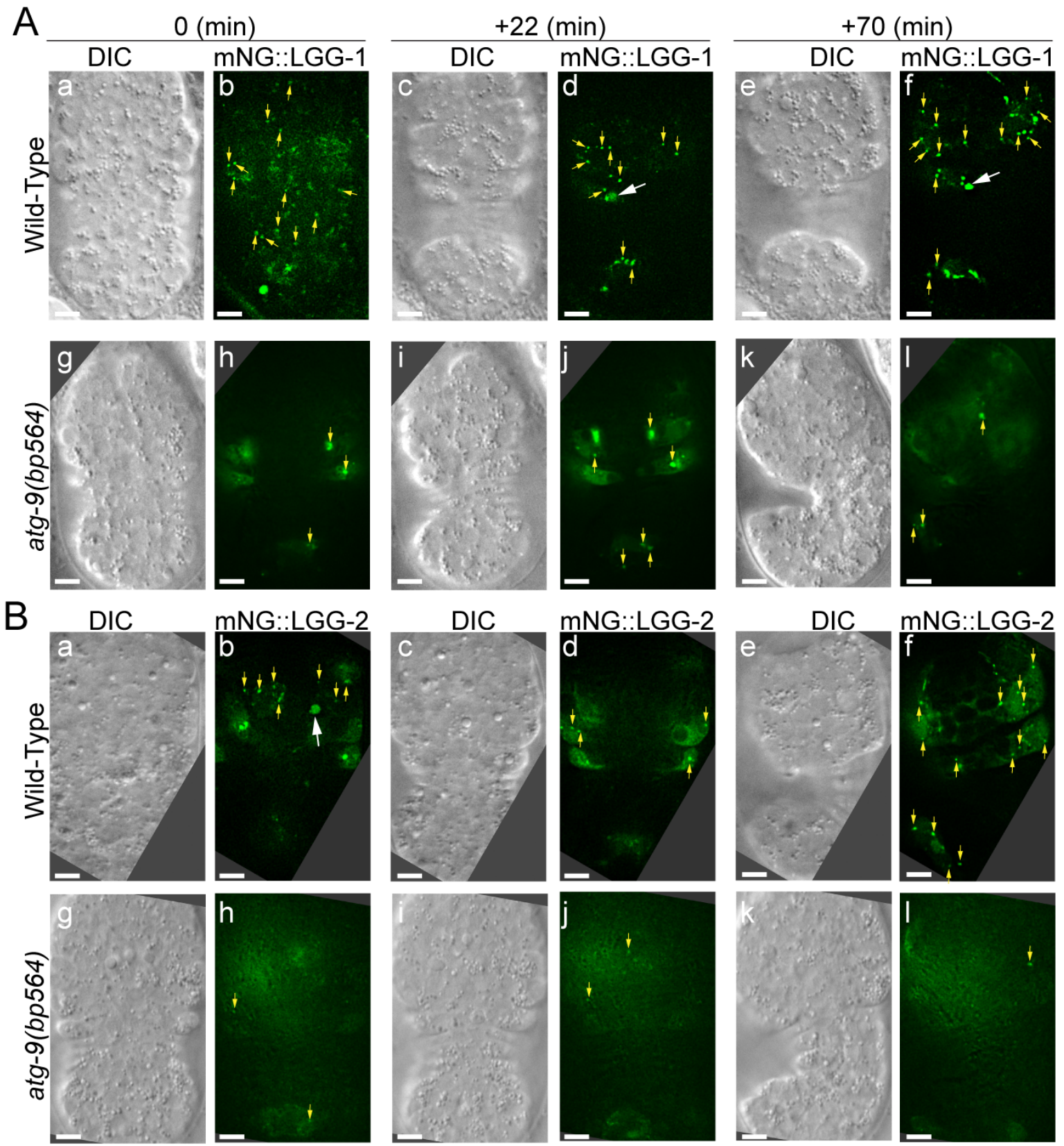

Figure S4. atg-9 is another gene essential for the production of autophagosomes and the

916 incorporation of autophagosomes into phagosomes

917 Images of wild-type and atg-9(bp564) mutant embryos expressing $\mathrm{P}_{c e d-1} m N G:: / g g-1$ (A) or ::Igg-2 (B)

918 are presented. " 0 min" embryos are embryos at $\sim 330$ min post $-1^{\text {st }}$ cleavage. The mNG images are $2-D$

919 projections of $14 \mathrm{Z}$-sections at $0.5 \mu \mathrm{m}$ intervals each. Green puncta (yellow arrows) represent

920 autophagosomes. White arrows in $(A(d, f))$ and $(B(b))$ mark phagosomes in which $m N G$ signal was

921 detected, indicating the existence of autophagosomes/phagosome fusion events. Scale bars are $5 \mu \mathrm{m}$. 
Figure S5. The expression of Igg-1 and Igg-2 cDNA in engulfing cells suppresses the Ced phenotype of Igg-1 and lgg-2 null mutants, respectively.

927 GFP- and mCherry-tagged reporters for LGG-1 and LGG-2 are expressed in engulfing cells under the 928 control of the $\mathrm{P}_{c e d-1}$ promoter in $\operatorname{lgg}$-1(tm3489) and $\operatorname{lgg}$-2(tm5755) mutant embryos, respectively. The 929 bar graph displays the average number of somatic cell corpses in lgg-1(tm3489) and lgg-2(tm5755) 930 mutant embryos at the 1.5-fold stage, both with and without the expression of the rescuing construct. 931 The number of embryos scored for each strain is in parentheses. Bars represent the mean, and the 932 error bars indicate standard deviation. Brackets above the bars indicate the samples that are 933 compared by the Student $t$-test. $p$-values are summarized as such: ${ }^{*}, 0.001<\mathrm{p}<0.05 ;{ }^{* *}, 0.0001<\mathrm{p}$ $934<0.001 ;{ }^{* *}, p<0.0001 ;$ ns, no significant difference. 
A

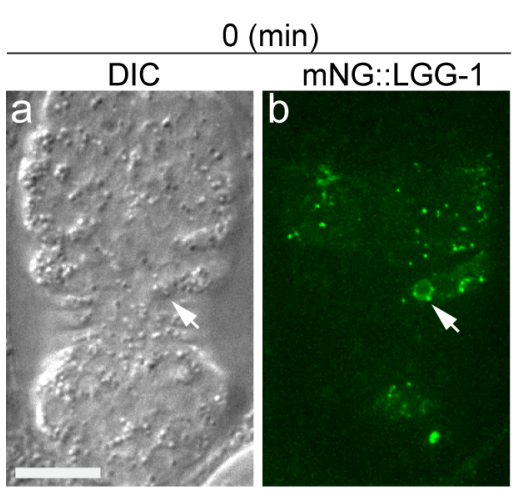

wild-type

ced-1

(e1735)
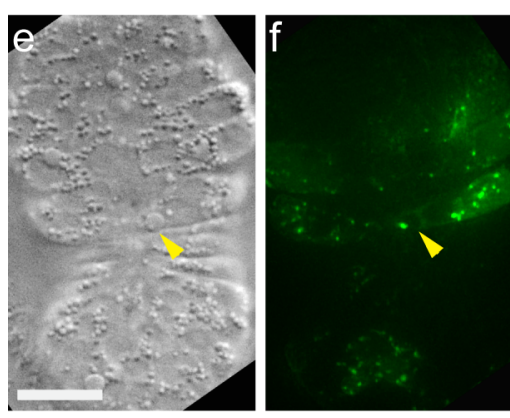

B

wild-type
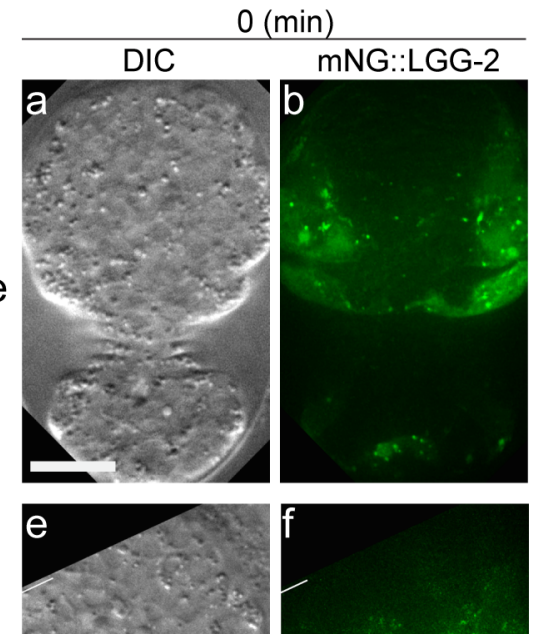

ced-1

(e1735)
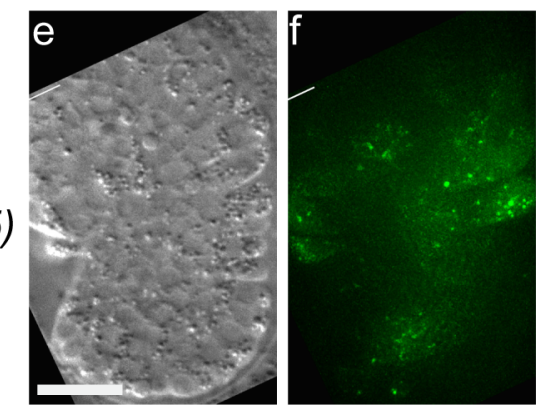
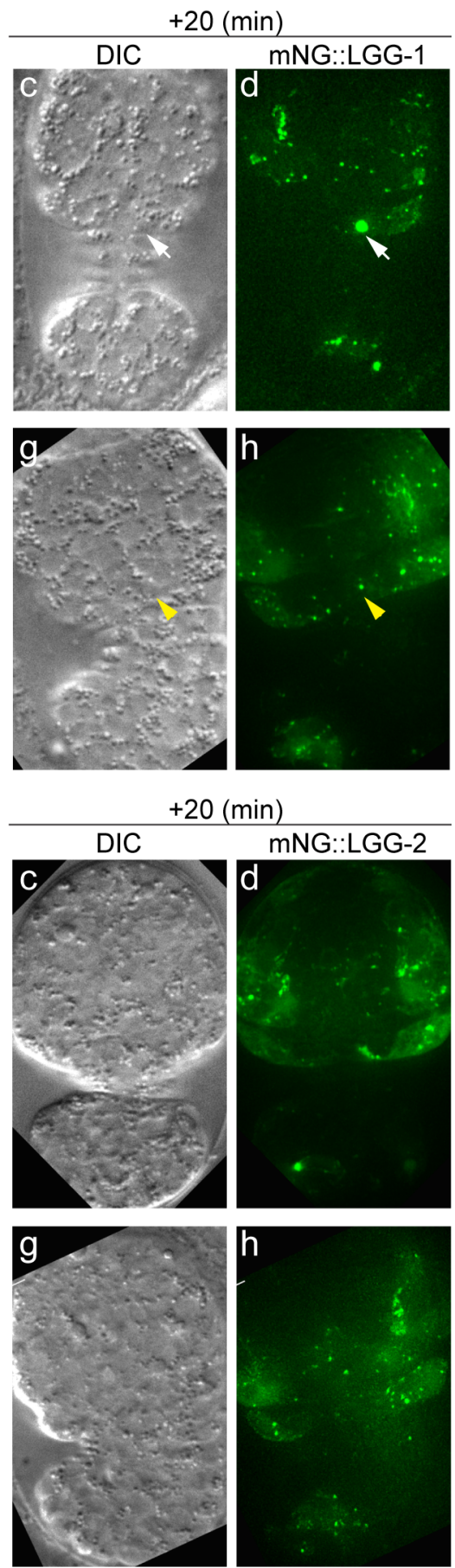

\section{Figure S6. The generation of autophagosomes is normal in ced-1 mutants.}

Images of wild-type and ced-1(e1735) mutant embryos expressing $\mathrm{P}_{c e d-1} m N G:: / g g-1(\mathrm{~A})$ or $\mathrm{P}_{\text {ced-1 }}$ lgg2::mNG (B) are presented. (a-b and e-f) Embryos are at $\sim 370$ min post- $1^{\text {st }}$ cleavage; (c-d and g-h) Embryos 20 min later. (a, c, e, g) DIC images. (b, d, f, h) 2-D projection of 14 consecutive Z-sections at $0.5 \mu \mathrm{m}$ interval each. Green puncta represent autophagosomes. White arrows in (A(a-d)) marks one C3 phagosome, onto which LGG-1::mNG puncta are recruited in (a-b); 20 min later (c-d), LGG$1:: \mathrm{mNG}$ enters this phagosome, showing an autophagosomes/phagosome fusion event. Yellow 
945 arrowheads in (A(e-h)) marks one C3 phagosome on the surface, of which only one mNG punctum was

946 found (f), and no mNG signal enters the phagosome (h), indicating the lack of recruitment and fusion.

947 Scale bars are $10 \mu \mathrm{m}$.

\section{Table S1.}

950

\begin{tabular}{|c|c|c|c|}
\hline $\begin{array}{l}\text { Reagent } \\
\text { Type }\end{array}$ & Designation & $\begin{array}{l}\text { Source or } \\
\text { Reference }\end{array}$ & Additional information \\
\hline E. coli & OP50 & CGC & OP50 \\
\hline C. elegans & N2 & CGC & Wild-type Bristol N2 \\
\hline C elegans & ZH0989 & [19] & $\begin{array}{l}\text { unc-76(e911) V enls36 [pUNC-76(+)(20ng/ul), } \\
\text { pZZ610(Pced-1 ced-1::gfp) (5ng/ml),plH1(Pced-1 } \\
\text { 2xFYVE::mRFP)(5ng/ul)] V }\end{array}$ \\
\hline C. elegans & $\mathrm{ZH} 2573$ & This study & $\begin{array}{l}\text { Igg-2(tm5755) IV ; unc-76(e911) V; enEx1223 [pXL89(Pced- } \\
\text { 1mcherry::Igg-2, 10ng/ul) + pUNC-76(25ng/ul)] }\end{array}$ \\
\hline C. elegans & $\mathrm{ZH} 2632$ & This study & $\begin{array}{l}\text { lgg-2(tm5755) IV; unc-76(e911) V; enEx1267 [Punc-76(+) } \\
\text { 20ng/ul, pTY02(Pced-1::gfp::Igg2)5ng/ul] }\end{array}$ \\
\hline C. elegans & $\mathrm{ZH} 2715$ & This study & $\begin{array}{l}\text { rab-7 (ok511) II / mln1 II ; unc-76(e911) V; enEx1320 I } \\
{[p U N C-76(+)(20 n g / u l), p X L 89(\text { Pced-1mCherry::Igg- }} \\
\text { 2) }(5 n g / u l)]\end{array}$ \\
\hline C. elegans & $\mathrm{ZH} 2782$ & This study & $\begin{array}{l}\text { rab-7 (ok511) II / mln1 II ; unc-76(e911) V; enEx1376 } \\
\text { [pUNC-76(+)(20ng/ul), pZZ1091(Pced-1 mCherry::Igg-1) } \\
(5 n g / u l)]\end{array}$ \\
\hline C. elegans & $\mathrm{ZH} 2831$ & This study & lgg-1(tm3489) II / mIn1 II ; Igg-2(tm5755) IV \\
\hline C. elegans & $\mathrm{ZH} 2835$ & This study & $\begin{array}{l}\text { lgg-1(tm3489) II / mIn1 II ; unc-76(e911) V enls36 [pUNC- } \\
76(+)(20 n g / u l), p Z Z 610(\text { Pced-1 ced-1::gfp) } \\
\text { (5ng/ul),plH1(Pced-1 2xFYVE::mRFP)(5ng/ul)] V }\end{array}$ \\
\hline C. elegans & ZH2838 & This study & $\begin{array}{l}\text { lgg-1(tm3489) II / mln1 II ; unc-76(e911) V; enEx1428 } \\
\text { [pUNC-76(+)(20ng/ul), pZZ1052(Pced-1 gfp::Igg-1) (5ng/ul))] }\end{array}$ \\
\hline C. elegans & $\mathrm{ZH} 2841$ & This study & $\begin{array}{l}\text { lgg-1(tm3489) II / mln1 II ; unc-76(e911) V; enEx1431 } \\
\text { [pUNC-76(+)(20ng/ul), pZZ1091(Pced-1 mCherry ::Igg-1) } \\
(5 n g / u l))]\end{array}$ \\
\hline C. elegans & $\mathrm{ZH} 2875$ & This study & $\begin{array}{l}\text { lgg-2(tm5755) IV; unc-76(e911) V enls36 [pUNC- } \\
\text { 76(+)(20ng/ul), pZZ610(Pced-1 ced-1::gfp) } \\
(5 n g / m l), p l H 1(P c e d-12 x F Y V E:: m R F P)(5 n g / u l)] V\end{array}$ \\
\hline C. elegans & ZH2889 & This study & $\begin{array}{l}\text { unc-76(e911) V; enEx1459 [pUNC-76 20ng/ul, pNL9(Pced-1 } \\
\text { PH::mrfp) (5ng/ul),(pOPR004)Pced-1 mNG::/gg-2 5ng/ul)] }\end{array}$ \\
\hline C. elegans & ZH2903 & This study & $\begin{array}{l}\text { atg-9(bp564) V him-5(e1490) V; lin-15AB(n765ts) X; } \\
\text { enEx1468 [Plin-15(+) 50ng/ul, pNL9(Pced-1 PH::mrfp) } \\
\text { (5ng/ul), pOPR007(Pced-1 mNG::/gg-1) (5ng/ul)] }\end{array}$ \\
\hline C. elegans & $\mathrm{ZH} 2907$ & This study & $\begin{array}{l}\text { vps-18(tm1125) II ; unc-76(e911) V; enls80 [pUNC- } \\
76(+)(20 n g / u l), p Z Z 985(P c e d-1 \text { ced-1::gfp) } \\
(5 n g / m l), p Z Z 1091(P c e d-1 m C h e r r y:: / g g-1)(5 n g / u l)] ~ I V\end{array}$ \\
\hline C. elegans & $\mathrm{ZH} 2916$ & This study & $\begin{array}{l}\text { ced-1(e1735) I; unc-76(e911) V; enEx1470 } \\
{[p O P R 007(P c e d-1 \text { mNG::lgg-1) (5ng/ul) + pUNC-76(+) }} \\
\text { (20ng/ul) + pNL9(Pced-1 PH::mrfp) (5ng/ul)] }\end{array}$ \\
\hline
\end{tabular}




\begin{tabular}{|c|c|c|c|}
\hline C. elegans & ZH2918 & This study & $\begin{array}{l}\text { unc-76(e911) V ; enls80 [pUNC-76(+)(20ng/ul), pZZ985(P } P_{\text {ced- }} \\
\text { 1 ced-1::gfp) (5ng/ml),pZZ1091(Pced-1mCherry::lgg- } \\
\text { 1)(5ng/ul)]IV }\end{array}$ \\
\hline C. elegans & $\mathrm{ZH} 2919$ & This study & $\begin{array}{l}\text { en/s82 [pUNC-76(+)(20ng/ul), pZZ985(Pced-1 ced-1::gfp) } \\
\text { (5ng/ml),pZZ1091(Pced-1mCherry::Igg-1)(5ng/ul)] II ; unc- } \\
\text { 76(e911) V }\end{array}$ \\
\hline C. elegans & ZH2921 & This study & $\begin{array}{l}\text { enls85[pUNC-76(+)(20ng/ul), pNL9(Pced-1 PH::mrfp) } \\
\text { (5ng/ml),pOPR004(Pced-1mNG::lgg-2)(5ng/ul)] V unc- } \\
\text { 76(e911) V. }\end{array}$ \\
\hline C. elegans & ZH2922 & This study & $\begin{array}{l}\text { atg-9(bp564) V him-5(e1490) V; lin-15AB(n765ts) X; } \\
\text { enEx1472 [Plin-15(+) 50ng/ul, pNL9(Pced-1 PH::mrfp) } \\
\text { (10ng/ul), (pOPR004) Pced-1 mNG::lgg-2 10ng/ul] }\end{array}$ \\
\hline C. elegans & $\mathrm{ZH} 2929$ & This study & $\begin{array}{l}\text { vps-18(tm1125) II ; unc-76(e911) V; enls83 [pUNC- } \\
76(+)(20 n g / u l), p Z Z 985(\text { Pced-1 ced-1::gfp) } \\
(5 n g / m l), p X L 89(\text { Pced-1mCherry::lgg-2)(5ng/ul)]X }\end{array}$ \\
\hline C. elegans & $\mathrm{ZH} 2934$ & This study & $\begin{array}{l}\text { ced-1(e1735) I; unc-76(e911) V enls85 [pUNC- } \\
76(+)(20 n g / u l), p N L 9(P c e d-1 P H:: m r f p) \\
\text { (5ng/ul),pOPR004(Pced-1mNG::lgg-2)(5ng/ul)] V }\end{array}$ \\
\hline C. elegans & $\mathrm{ZH} 2950$ & This study & $\begin{array}{l}\text { enls82 [pUNC-76(+)(20ng/ul), pZZ985(Pced }-1 \text { ced-1::gfp) } \\
\text { (5ng/ml),pZZ1091(Pced-1mCherry::lgg-1)(5ng/ul)] II ; atg- } \\
\text { 7(bp411) IV; unc-76(e911) V }\end{array}$ \\
\hline C. elegans & $\mathrm{ZH} 2951$ & This study & $\begin{array}{l}\text { atg-7(bp411) IV; en/s83 [pUNC-76(+)(20ng/ul), } \\
\text { pZZ985(Pced-1 ced-1::gfp) (5ng/ml),pXL89(Pced- } \\
1 m \text { Cherry::lgg-2)(5ng/ul)] X }\end{array}$ \\
\hline C. elegans & $\mathrm{ZH} 2952$ & This study & $\begin{array}{l}\text { enls82 [pUNC-76(+)(20ng/ul), pZZ985(Pced-1 ced-1::gfp) } \\
\text { (5ng/ml),pZZ1091(Pced-1mCherry::lgg-1)(5ng/ul)] II ; atg- } \\
\text { 13(bp414) III }\end{array}$ \\
\hline C. elegans & $\mathrm{ZH} 2953$ & This study & $\begin{array}{l}\text { atg-13(bp414) III ; enls83 [pUNC-76(+)(20ng/ul), } \\
\text { pZZ985(Pced-1 ced-1::gfp) (5ng/ml),pXL89(Pced- } \\
1 m \text { Cherry::lgg-2)(5ng/ul)]X }\end{array}$ \\
\hline C. elegans & $\mathrm{ZH} 2954$ & This study & $\begin{array}{l}\text { epg-8(bp251) I; enls82 [pUNC-76(+)(20ng/ul), } \\
\text { pZZ985(Pced-1 ced-1::gfp) (5ng/ul),pZZ1091(Pced- } \\
1 m \text { Cherry::Igg-1)(5ng/ul)] II ; unc-76(e911) V }\end{array}$ \\
\hline C. elegans & $\mathrm{ZH} 2955$ & This study & $\begin{array}{l}\text { epg-8(bp251) I; unc-76(e911) him-5(1490) V; enls83 } \\
\text { [pUNC-76(+)(20ng/ul), pZZ985(Pced-1 ced-1::gfp) } \\
\text { (5ng/ul),pXL89(Pced-1mCherry::lgg-2)(5ng/ul)]X }\end{array}$ \\
\hline C. elegans & $\mathrm{ZH} 2980$ & This study & $\begin{array}{l}\text { atg-7(bp411) IV; enls36 [pUNC-76(+)(20ng/ul), } \\
\text { pZZ610(Pced-1 ced-1::gfp) (5ng/ml),plH1(Pced-1 } \\
\text { 2xFYVE::mRFP)(5ng/ul)] V unc-76(e911) V }\end{array}$ \\
\hline C. elegans & ZH2992 & This study & $\begin{array}{l}\text { enls87 [pUNC-76 20ng/ul, pNL9(Pced-1 PH::mrfp) (5ng/ul), } \\
\text { pOPR007(Pced-1 mNG::lgg-1) (5ng/ul)]l ; unc-76(e911) V }\end{array}$ \\
\hline C. elegans & ZH2994 & This study & $\begin{array}{l}\text { en/s82 [pUNC-76(+)(20ng/ul), pZZ985(Pced-1 ced-1::gfp) } \\
(5 n g / m l), p Z Z 1091(P c e d-1 m C h e r r y:: l g g-1)(5 n g / u l)] ~ I I ; \text { ced- } \\
6(n 2095) \text { III ; unc-76(e911) V }\end{array}$ \\
\hline C. elegans & ZH2995 & This study & $\begin{array}{l}\text { ced-6(n2095) III ; unc-76(e911) V; enls83 [pUNC- } \\
76(+)(20 n g / u l), p Z Z 985(P c e d-1 \text { ced-1::gfp) } \\
(5 n g / m l), p X L 89(\text { Pced-1mCherry::lgg-2)(5ng/ul)]X }\end{array}$ \\
\hline C. elegans & ZH3009 & This study & $\begin{array}{l}\text { ced-5(n1812) IV ; unc-76(e911) V; enls83 [pUNC- } \\
76(+)(20 n g / u l), \text { pZZ985(Pced-1 ced-1::gfp) } \\
(5 n g / m l), p X L 89(\text { Pced-1mCherry::Igg-2)(5ng/ul)]X }\end{array}$ \\
\hline C. elegans & $\mathrm{ZH} 3010$ & This study & enIs82 II ; ced-5(n1812) IV; unc-76(e911) V \\
\hline
\end{tabular}




\begin{tabular}{|l|l|l|l|}
\hline C. elegans & ZH3011 & This study & en/s82 II ; ced-10(n1993) IV; unc-76(e911) V \\
\hline C. elegans & ZH3012 & This study & $\begin{array}{l}\text { ced-10(n1993) IV; unc-76(e911) V; en/s83 [pUNC- } \\
76(+)(20 n g / u l), p Z Z 985(P c e d-1 \text { ced-1::gfp) } \\
(5 n g / m l), p X L 89(P c e d-1 m C h e r r y:: I g g-2)(5 n g / u l)] X\end{array}$ \\
\hline C. elegans & ZH3014 & This study & $\begin{array}{l}\text { en/s87 I ; unc-76(e911) V; dyn-1(n4039) X; enEx21[Pdyn-1 } \\
\text { dyn-1 8ng/ul)] }\end{array}$ \\
\hline C. elegans & ZH3015 & This study & $\begin{array}{l}\text { en/s85 V unc-76(e911) V;dyn-1(n4039) X; enEx21[Pdyn-1 } \\
\text { dyn-1 8ng/ul)] }\end{array}$ \\
\hline
\end{tabular}

\section{Supplemental movie legends}

\section{Movie S1. mCherry::LGG-1-labeled vesicles are recruited to the surface of a phagosome and} subsequently fuse to the phagosome

Related to Fig 1E. This movie shows time-lapse recording images of a C3 phagosome (white arrowheads) in a wild-type embryo, starting at $\sim 330$ min post- $1^{\text {st }}$ cleavage. CED-1::GFP labels pseudopods, allowing the visualization of the phagosome formation process. " $0 \mathrm{~min}$ " is the moment when pseudopods just seals. Yellow arrowheads indicate the mCherry::LGG-1 ${ }^{+}$puncta, and open white

Movie S2. mCherry::LGG-2-labeled vesicles are recruited to the surface of a phagosome and subsequently fuse to the phagosome

Related to Fig 1H. This movie shows time-lapse recording images of a C3 phagosome (white arrowheads) in a wild-type embryo, starting at $\sim 330$ min post $-1^{\text {st }}$ cleavage. CED-1::GFP labels pseudopods, allowing the visualization of the phagosome formation process. " $0 \mathrm{~min}$ " is the moment when pseudopods just seals. Yellow arrowheads indicate the mCherry::LGG-2 ${ }^{+}$puncta, and open white arrows point to the phagosome lumen containing the mCherry signal.

Movie S3. mNG::LGG-1-labeled vesicles are recruited to the surface of a phagosome and subsequently fuse to the phagosome.

Related to Fig 1F. This movie shows time-lapse recording images of a C3 phagosome in a wild-type embryo, starting at $\sim 330$ min post- $1^{\text {st }}$ cleavage. $\mathrm{PH}(\mathrm{hPLCY}):: \mathrm{mRFP}$ labels pseudopods, allowing the visualization of the phagosome formation process. " $0 \mathrm{~min}$ " is the moment when pseudopods just seals. Yellow arrowheads indicate $\mathrm{mNG}:: \mathrm{LGG}-1^{+}$puncta, and open white arrows point to the phagosome with $\mathrm{mNG}$ signal in its lumen.

Movie S4. In rab-7 (ok511), mCherry::LGG-1-labeled vesicles fail to fuse with phagosomes.

Related to Fig 7E. This movie shows time-lapse recording images of a C3 phagosome in a rab- 
981 a nascent phagosome is just formed. White arrowheads mark the phagosome. Yellow arrows mark

982 mCherry::LGG-1+ $1^{+}$puncta.

983

984 Movie S5. In rab-7(ok511) mutants, mCherry::LGG-2-labeled vesicles fail to fuse with 985 phagosomes.

986 Related to Fig 7F. This movie shows time-lapse recording images of a C3 phagosome in a rab987 7(ok511) m־z homozygous embryos, starting at $\sim 330$ min post- $1^{\text {st }}$ cleavage. " 0 min" is the moment 988 when a nascent phagosome is just formed. White arrowheads mark the phagosome. Yellow arrows 989 mark mCherry::LGG-2+ puncta.

990

991 Movie S6. In ced-1(e1735) mutants, mNG::LGG-1-labeled vesicles fail to be recruited to the 992 phagosomal surface.

993 Related to Fig 8A. This movie shows time-lapse recording images of a C3 phagosome in a ced994 1(e1735) embryo, starting at 200 min post $-1^{\text {st }}$ cleavage. " 0 min" is the moment when a nascent 995 phagosome is just formed. White arrowheads mark the phagosome; yellow arrows label mNG::LGG-1 ${ }^{+}$ 996 puncta.

997 


\section{References}

999 1. Reddien PW, Horvitz HR (2004) The engulfment process of programmed cell death in caenorhabditis elegans. Annu Rev Cell Dev Biol 20: 193-221.

1001 2. Nagata S (2018) Apoptosis and Clearance of Apoptotic Cells. Annu Rev Immunol.

1002 3. Levin R, Grinstein S, Canton J (2016) The life cycle of phagosomes: formation, maturation, and 1003 resolution. Immunol Rev 273: 156-179.

1004 4. Schaaf MB, Keulers TG, Vooijs MA, Rouschop KM (2016) LC3/GABARAP family proteins: 1005 autophagy-(un)related functions. FASEB J 30: 3961-3978.

5. Sanjuan MA, Dillon CP, Tait SW, Moshiach S, Dorsey F, et al. (2007) Toll-like receptor signalling in macrophages links the autophagy pathway to phagocytosis. Nature 450: 1253-1257.

6. Green DR, Oguin TH, Martinez J (2016) The clearance of dying cells: table for two. Cell Death Differ 23: 915-926.

7. Martinez J, Almendinger J, Oberst A, Ness R, Dillon CP, et al. (2011) Microtubule-associated protein 1 light chain 3 alpha (LC3)-associated phagocytosis is required for the efficient clearance of dead cells. Proc Natl Acad Sci U S A 108: 17396-17401.

8. Martinez J, Cunha LD, Park S, Yang M, Lu Q, et al. (2016) Noncanonical autophagy inhibits the autoinflammatory, lupus-like response to dying cells. Nature 533: 115-119.

9. Morishita H, Mizushima N (2019) Diverse Cellular Roles of Autophagy. Annu Rev Cell Dev Biol 35 : 453-475.

10. Nakatogawa H (2020) Mechanisms governing autophagosome biogenesis. Nat Rev Mol Cell Biol 21: 439-458.

11. Cheng S, Wu Y, Lu Q, Yan J, Zhang H, et al. (2013) Autophagy genes coordinate with the class II

12. Huang S, Jia K, Wang Y, Zhou Z, Levine B (2013) Autophagy genes function in apoptotic cell corpse clearance during C. elegans embryonic development. Autophagy 9: 138-149.

13. Li W, Zou W, Yang Y, Chai Y, Chen B, et al. (2012) Autophagy genes function sequentially to promote apoptotic cell corpse degradation in the engulfing cell. J Cell Biol 197: 27-35. Nat Cell Biol 17: 893-906. 
1029 15. Sulston JE, Horvitz HR (1977) Post-embryonic cell lineages of the nematode, Caenorhabditis 1030 elegans. Dev Biol 56: 110-156.

1031 16. Sulston JE, Albertson DG, Thomson JN (1980) The Caenorhabditis elegans male: postembryonic 1032 development of nongonadal structures. Dev Biol 78: 542-576.

1033 17. Ellis RE, Jacobson DM, Horvitz HR (1991a) Genes required for the engulfment of cell corpses 1034 during programmed cell death in Caenorhabditis elegans. Genetics 129: 79-94.

1035 18. Mangahas PM, Zhou Z (2005) Clearance of apoptotic cells in Caenorhabditis elegans. Semin Cell 1036 Dev Biol 16: 295-306.

1037 19. Yu X, Lu N, Zhou Z (2008) Phagocytic receptor CED-1 initiates a signaling pathway for degrading 1038 engulfed apoptotic cells. PLoS Biol 6: e61.

1039 20. Zhou Z, Hartwieg E, Horvitz HR (2001b) CED-1 is a transmembrane receptor that mediates cell $1040 \quad$ corpse engulfment in C. elegans. Cell 104: 43-56.

1041 21. Yu X, Odera S, Chuang CH, Lu N, Zhou Z (2006) C. elegans Dynamin mediates the signaling of 1042 phagocytic receptor CED-1 for the engulfment and degradation of apoptotic cells. Dev Cell 10: 743-757.

22. Shen Q, He B, Lu N, Conradt B, Grant BD, et al. (2013) Phagocytic receptor signaling regulates clathrin and epsin-mediated cytoskeletal remodeling during apoptotic cell engulfment in C. elegans. Development 140: 3230-3243.

23. Lu N, Shen Q, Mahoney TR, Neukomm LJ, Wang Y, et al. (2012) Two PI 3-kinases and one PI 3phosphatase together establish the cyclic waves of phagosomal PtdIns(3)P critical for the degradation of apoptotic cells. PLoS Biol 10: e1001245.

25. Lu N, Zhou Z (2012) Membrane trafficking and phagosome maturation during the clearance of apoptotic cells. Int Rev Cell Mol Biol 293: 269-309.

26. Manil-Segalen M, Lefebvre C, Jenzer C, Trichet M, Boulogne C, et al. (2014) The C. elegans LC3 acts downstream of GABARAP to degrade autophagosomes by interacting with the HOPS subunit VPS39. Dev Cell 28: 43-55. nematode Caenorhabditis elegans. Methods Mol Biol 559: 357-370. 
1059 28. Wu F, Watanabe Y, Guo XY, Qi X, Wang P, et al. (2015) Structural Basis of the Differential Function of the Two C. elegans Atg8 Homologs, LGG-1 and LGG-2, in Autophagy. Mol Cell 60: 914-929.

29. Tsien RY (1998) The green fluorescent protein. Annu Rev Biochem 67: 509-544.

1063

1064

1065

1066

1067

1068

1069

1070

1071

1072

1073

1074

1075

1076

1077

1078

1079

1080

1081

1082

1083

1084

1085

1086

1087

1088

1089

30. Shaner NC, Campbell RE, Steinbach PA, Giepmans BN, Palmer AE, et al. (2004) Improved monomeric red, orange and yellow fluorescent proteins derived from Discosoma sp. red fluorescent protein. Nat Biotechnol 22: 1567-1572.

31. Shinoda H, Ma Y, Nakashima R, Sakurai K, Matsuda T, et al. (2018) Acid-Tolerant Monomeric GFP from Olindias formosa. Cell Chem Biol 25: 330-338 e337.

32. Tian Y, Li Z, Hu W, Ren H, Tian E, et al. (2010) C. elegans screen identifies autophagy genes specific to multicellular organisms. Cell 141: 1042-1055.

33. Yang P, Zhang H (2011) The coiled-coil domain protein EPG-8 plays an essential role in the autophagy pathway in C. elegans. Autophagy 7: 159-165.

34. Zhang H, Baehrecke EH (2015) Eaten alive: novel insights into autophagy from multicellular model systems. Trends Cell Biol.

35. Gomes LC, Odedra D, Dikic I, Pohl C (2016) Autophagy and modular restructuring of metabolism control germline tumor differentiation and proliferation in C. elegans. Autophagy 12: 529-546.

36. Lin L, Yang P, Huang X, Zhang H, Lu Q, et al. (2013) The scaffold protein EPG-7 links cargoreceptor complexes with the autophagic assembly machinery. J Cell Biol 201: 113-129.

37. Lu Q, Yang P, Huang X, Hu W, Guo B, et al. (2011) The WD40 repeat PtdIns(3)P-binding protein EPG-6 regulates progression of omegasomes to autophagosomes. Dev Cell 21: 343-357.

38. Wu F, Wang P, Shen Y, Noda NN, Zhang H (2016) Small differences make a big impact: Structural insights into the differential function of the 2 Atg8 homologs in C. elegans. Autophagy 12: 606607.

39. Balderhaar HJ, Ungermann C (2013) CORVET and HOPS tethering complexes - coordinators of endosome and lysosome fusion. J Cell Sci 126: 1307-1316.

40. Szatmari Z, Sass M (2014) The autophagic roles of Rab small GTPases and their upstream regulators: a review. Autophagy 10: 1154-1166.

41. McEwan DG, Popovic D, Gubas A, Terawaki S, Suzuki H, et al. (2015) PLEKHM1 regulates autophagosome-lysosome fusion through HOPS complex and LC3/GABARAP proteins. Mol Cell 57: 39-54. 
1090 42. Xiao H, Chen D, Fang Z, Xu J, Sun X, et al. (2009) Lysosome biogenesis mediated by vps-18 affects apoptotic cell degradation in Caenorhabditis elegans. Mol Biol Cell 20: 21-32.

1092 43. Wu YC, Stanfield GM, Horvitz HR (2000) NUC-1, a caenorhabditis elegans DNase II homolog, functions in an intermediate step of DNA degradation during apoptosis. Genes Dev 14: 536-548.

44. Guo P, Hu T, Zhang J, Jiang S, Wang X (2010) Sequential action of Caenorhabditis elegans Rab GTPases regulates phagolysosome formation during apoptotic cell degradation. Proc Natl Acad Sci U S A 107: 18016-18021.

45. Qu X, Zou Z, Sun Q, Luby-Phelps K, Cheng P, et al. (2007) Autophagy gene-dependent clearance of apoptotic cells during embryonic development. Cell 128: 931-946.

46. Jenzer C, Simionato E, Largeau C, Scarcelli V, Lefebvre C, et al. (2019) Autophagy mediates phosphatidylserine exposure and phagosome degradation during apoptosis through specific functions of GABARAP/LGG-1 and LC3/LGG-2. Autophagy 15: 228-241.

47. Fazeli G, Stetter M, Lisack JN, Wehman AM (2018) C. elegans Blastomeres Clear the Corpse of the Second Polar Body by LC3-Associated Phagocytosis. Cell Rep 23: 2070-2082.

48. Fazeli G, Trinkwalder M, Irmisch L, Wehman AM (2016) C. elegans midbodies are released, phagocytosed and undergo LC3-dependent degradation independent of macroautophagy. J Cell Sci 129: 3721-3731.

49. Vieira OV, Botelho RJ, Grinstein S (2002) Phagosome maturation: aging gracefully. Biochem J 366: 689-704.

50. Spang A (2016) Membrane Tethering Complexes in the Endosomal System. Front Cell Dev Biol 4:

1114 52. Jiang P, Nishimura T, Sakamaki Y, Itakura E, Hatta T, et al. (2014) The HOPS complex mediates 1115 autophagosome-lysosome fusion through interaction with syntaxin 17. Mol Biol Cell 25: 1327$1116 \quad 1337$.

1117 53. Takáts S, Pircs K, Nagy P, Varga Á, Kárpáti M, et al. (2014) Interaction of the HOPS complex with 1118 Syntaxin 17 mediates autophagosome clearance in Drosophila. Mol Biol Cell 25: 1338-1354. 1119 54. Stroupe C, Collins KM, Fratti RA, Wickner W (2006) Purification of active HOPS complex reveals its affinities for phosphoinositides and the SNARE Vam7p. EMBO J 25: 1579-1589. 
1121 55. Jeschke A, Haas A (2018) Sequential actions of phosphatidylinositol phosphates regulate phagosome-lysosome fusion. Mol Biol Cell 29: 452-465.

1123 56. Wood WB, Researchers tCoCe (1988) The Nematode Caenorhabditis elegans. Cold Spring Harbor, $1124 \quad$ NY: Cold Spring Harbor Laboratory.

1125 57. Riddle DL, Blumenthal T, Meyer BJ, Priess JR, editors (1997) C. elegans II. Plainview, NY: Cold 1126 Spring harbor Laboratory Press.

1127 58. Edgley ML, Baillie DL, Riddle DL, Rose AM (2006) Genetic balancers. WormBook: 1-32.

1128 59. Bloom L, Horvitz HR (1997) The Caenorhabditis elegans gene unc-76 and its human homologs

1129 define a new gene family involved in axonal outgrowth and fasciculation. Proc Natl Acad Sci U 1130 S A 94: 3414-3419.

1131 60. Jin Y (1999) Transformation. In: Hope IA, editor. C elegans, a practical approach. Oxford: Oxford 1132 University Press. pp. 69-96.

1133 61. Haley R, Wang Y, Zhou Z (2018) The small GTPase RAB-35 defines a third pathway that is 1134 required for the recognition and degradation of apoptotic cells. PLoS Genet 14: e1007558.

1135 62. Shaner NC, Lambert GG, Chammas A, Ni Y, Cranfill PJ, et al. (2013) A bright monomeric green 1136 fluorescent protein derived from Branchiostoma lanceolatum. Nat Methods 10: 407-409. 